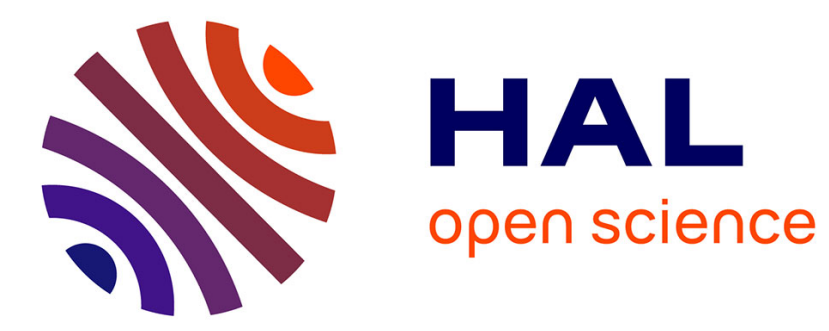

\title{
About a stability conjecture concerning unilateral contact with friction
}

Elaine Pratt, Alain Léger, Michel Jean

\section{To cite this version:}

Elaine Pratt, Alain Léger, Michel Jean. About a stability conjecture concerning unilateral contact with friction. Nonlinear Dynamics, 2010, 59 (1-2), pp.73-94. 10.1007/s11071-009-9522-z . hal-01580814

\section{HAL Id: hal-01580814 \\ https://hal.science/hal-01580814}

Submitted on 4 Sep 2017

HAL is a multi-disciplinary open access archive for the deposit and dissemination of scientific research documents, whether they are published or not. The documents may come from teaching and research institutions in France or abroad, or from public or private research centers.
L'archive ouverte pluridisciplinaire HAL, est destinée au dépôt et à la diffusion de documents scientifiques de niveau recherche, publiés ou non, émanant des établissements d'enseignement et de recherche français ou étrangers, des laboratoires publics ou privés. 


\title{
About a stability conjecture concerning unilateral contact with friction
}

\author{
Elaine Pratt • Alain Léger · Michel Jean
}

\author{
E. Pratt \\ Centre de Mathématiques et d'Informatique, Aix-Marseille \\ Université, 39, rue F. Joliot Curie, 13453 Marseille Cedex \\ 13, France \\ e-mail: pratt@lma.cnrs-mrs.fr
}

\author{
A. Léger · M. Jean \\ Laboratoire de Mécanique et d'Acoustique, CNRS, 31, \\ chemin Joseph Aiguier, 13402 Marseille Cedex 20, France
}

\begin{abstract}
A new notion of stability specially adapted to systems with unilateral contact and Coulomb friction is introduced. Whereas classical stability results in mechanics concern perturbations of the initial data in a classical phase space, we establish here results concerning the trajectories issued from a perturbation of the external forces. In such a context we state a conjecture concerning stability with respect to external forces that we back up by analytical computations in the case of simple models and then by numerical computations for more complex systems.
\end{abstract}

Keywords Coulomb friction · Unilateral contact · Nonsmooth dynamics · Mass-spring systems . Stability

\section{Introduction}

This paper aims at revisiting the basic stability concepts in the case of equilibrium states of discrete sys- tems involving unilateral contact and Coulomb friction. The inequalities induced by the contact and the friction laws, in addition to the dissipative character of Coulomb friction, make it impossible to use the classical stability theorems of discrete systems such as the Lejeune Dirichlet theorem. Moreover, the graphs of the contact and friction laws rule out any linearization.

Within such a framework, the present work follows recent papers in which stability properties were obtained by a direct integration of the dynamics [3, 5]. Let us recall that these analyses consisted in perturbing an equilibrium state in a classical phase space and then calculating the evolution in time of the distance between the initial equilibrium state and the trajectory having the perturbed state as initial data. If any neighborhood of the equilibrium contains a point which, taken as initial data of the dynamical problem, leads to a trajectory which diverges from the equilibrium, then the equilibrium is said to be unstable. On the contrary, if taking any point of a neighborhood of the equilibrium as initial data one gets a trajectory which tends to the equilibrium or remains in a tubular neighborhood of the equilibrium, then the equilibrium is said to be asymptotically stable, or stable in the sense of Lyapunov.

It is clear, and it has been stressed in the works mentioned above, that such an analysis can be undergone only after having proved that: 
- The set of equilibria is completely determined for any set of external data (loads, stiffness, friction coefficient, etc.).

- The Cauchy problem is well-posed (which means that the problem consisting in the equation of the dynamics associated with any admissible initial data has a unique solution).

- The solution of a discretized problem converges, as the time step tends to zero, towards the solution of the Cauchy problem.

Of course the fact that the set of equilibria for given forces consists in a single point, a set of discrete points, or a continuous set indeed even an unbounded one, has an effect on the stability properties. This was initially brought to light for a particular case in [10]. Note that although the existence of a trajectory can hold under very large conditions, only very smooth data ensures its uniqueness [4]. The estimates on the distance between a given trajectory and an equilibrium are generally obtained through a discretized problem, so that the convergence result is essential. But in fact:

- The complexity of the problem, simply investigating and classifying the equilibrium states, increases rapidly with the number of degrees of freedom of the system, so that the program consisting in the above three steps has for the moment only been tackled for a mass-spring system containing only one particle.

- The elementary and classical notion of stability which justifies the analysis may not seem totally satisfactory in view of the graph of the Coulomb law. Indeed, an equilibrium solution can be perturbed by a tangential velocity only if it is in imminent sliding. Which means that a given strictly stuck equilibrium solution can be perturbed by a tangential velocity only after the reaction has jumped to the edge of the Coulomb cone, so that even for very small velocities the modification of the reaction may have to be extremely large. This means in turn that it is quite possible that an equilibrium defined by ( $U=U^{\mathrm{eq}}, \dot{U}=0$ ) is not modified by adding any relatively small external force. Indeed, the deeper are the reactions inside the Coulomb cone, the larger the perturbation may have to be.

The present paper is a contribution to back up a conjecture which results from the observation of a large number of numerical experiments. A new notion of stability related to the external forces which has been suggested recently in [2] is needed. We first observe that it is equivalent to say that an equilibrium ( $U=U^{\mathrm{eq}}, \dot{U}=0$ ) is not perturbed by a small enough external force or to say that the corresponding reaction is strictly inside the Coulomb cone. Then the conjecture can be qualitatively formulated in the following way:

Conjecture Let a discrete system with any finite number of degrees of freedom be submitted to unilateral contact and Coulomb friction. Assume the data are such that there exists an equilibrium state in which some reactions are strictly inside the Coulomb cone while the other reactions are in imminent sliding and no reactions are in grazing contact. Then the trajectory produced by any sufficiently small perturbation of the data leads to a new equilibrium where the number of reactions strictly inside the cone is larger than before the perturbation.

This statement of the conjecture concerns any type of finite dimensional system with unilateral contact and Coulomb friction that means both granular media, i.e. collections of rigid bodies without any stiffness matrix, and systems having a nonzero stiffness matrix. In the present work we restrict our attention to mass-spring systems, i.e. with a nonzero stiffness matrix.

As a corollary of the above conjecture we shall observe in the case of a nonzero stiffness matrix that if the perturbing force does not depend on time then the final equilibrium is reached in finite time and all the reactions are strictly inside the Coulomb cone.

We now outline the main parts of the paper.

In the first section we make sure that the conjecture is in agreement with the behavior of a few quite simple models. In the case where the normal components of the reactions are given, we simply show that it is always possible to find a sufficiently small perturbation satisfying the above conjecture. This just amounts to revisiting some previous calculations concerning the motion of a mass-spring chain moving on a horizontal plane and submitted to gravity and to horizontal perturbations [13]. Going on with simple models the second part of this section deals with the so-called Klarbring's model [9]. Extending some previous calculations to the case when the external loading is timedependent, we show that the dynamics of this simple system with a strict Coulomb friction law is also in agreement with the conjecture.

In the second section we proceed in the justification of the conjecture from the point of view of analyti- 
cal calculations of the dynamics of simple models. We consider a mass-spring system which is more or less built by coupling two Klarbring's models. It appears that, even though the system is just slightly more complicated than Klarbring's model, two masses submitted to unilateral contact and Coulomb friction instead of only one, the set of equilibrium states under given forces is by far more intricate, and a complete explicit analysis of this set is required in order to undergo the following analysis. In the next part of this section we consider equilibrium states where either one or two masses are in imminent slip and we show that in all cases, adding a sufficiently small loading brings the system into a strictly stuck equilibrium state.

We then conclude the paper by computing the perturbation of an elastic bloc which has been discretized by finite elements and where a number of contact nodes are in imminent sliding. A specific postprocessing shows how the number of reactions strictly inside the cone increase if the perturbations are small enough.

\section{Analysis of simple models}

Let us write the equations of the dynamics of any discrete system in the following abstract form:

$$
\left\{\begin{array}{l}
\ddot{u}+K u=F+R, \\
+ \text { Unilateral contact, } \\
\text { + Coulomb friction, } \\
\text { +Impact law, } \\
\text { + Initial data, }
\end{array}\right.
$$

so that the equilibrium equations are:

$$
\left\{\begin{array}{l}
K u=F+R, \\
+ \text { Unilateral contact, } \\
\text { +Coulomb friction. }
\end{array}\right.
$$

The Coulomb friction law implies that a particle can be set into motion only if its reaction reaches the border of the Coulomb cone. So let an equilibrium state be determined by a pair $(u, R)$ where $R$ is strictly inside the Coulomb cone, then (2) show that the external forces $F$ can be changed without producing any motion as long as the corresponding reaction $R$ remains strictly inside the cone. This specific property of the equilibrium solutions, which is due to Coulomb friction, shall be developed in the following sections and leads to the following definition:

Definition A solution $(u(t), R(t))$ to problem (1) where $u(t)=u_{0}=$ constant is called a space equilibrium.

We stress the fact that throughout this paper we use the non-regularized Coulomb law expressed at a contact point by:

$$
\begin{aligned}
& \left|R_{t}\right| \leq \mu R_{n} \quad \text { and } \\
& \left\{\begin{array}{l}
\left|R_{t}\right|<\mu R_{n} \Longrightarrow \dot{u}_{t}=0, \\
\left|R_{t}\right|=\mu R_{n} \Longrightarrow \exists \lambda \geq 0 \text { such that } \dot{u}_{t}=-\lambda R_{t} .
\end{array}\right.
\end{aligned}
$$

$R_{t}$ and $R_{n}$ are respectively the tangential and the normal components of the reaction to the obstacle, $\mu$ is the friction coefficient, $u_{t}$ the tangential displacement and $(\cdot)$ stands for the time derivative.

\subsection{Where the normal reaction is given}

We consider here a mass-spring chain moving on a horizontal line submitted to gravity and to horizontal perturbations in the direction of the line, so that the motion is unidimensional. This system was extensively studied in [13] in another context and we shall simply recall below the results relevant to the conjecture.

A constant driving force is applied so that the solution is both unique and sufficiently smooth (see [6]). Figure 1 represents the reference configuration.

We begin by considering two masses linked together by a spring and moving on a plane with Coulomb friction.

The equations governing the motion in the case of two masses, after a rescaling which involves the mass, the stiffness of the spring and the friction coefficient, are the following:

$$
\left\{\begin{array}{l}
\ddot{u}_{1}+\left(u_{1}-u_{2}\right)=r_{1}+\varepsilon, \quad t>0 \\
\ddot{u}_{2}+\left(u_{2}-u_{1}\right)=r_{2}, \\
-1 \leq r_{1} \leq+1, \quad-1 \leq r_{2} \leq+1 \\
u_{1}(0)=-1, \quad u_{2}(0)=0 \\
\dot{u}_{1}(0)=\dot{u}_{2}(0)=0 \\
r_{1}(0)=-1, \quad r_{2}(0)=+1
\end{array}\right.
$$


Fig. 1 A chain of $n$ masses

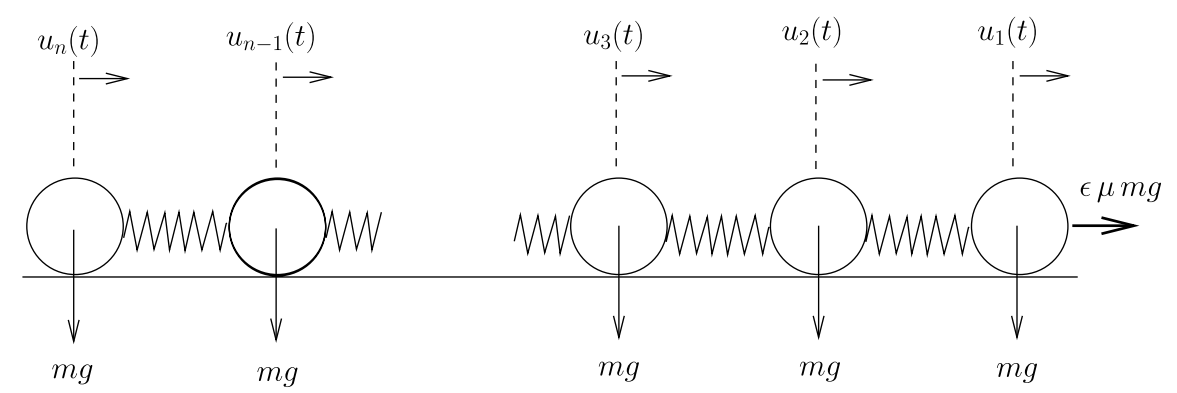

where $r_{1}$ and $r_{2}$ are the rescaled tangential components of the reaction (i.e. $r_{1}=\frac{R_{1}}{\mu m g}$ and $r_{2}=\frac{R_{2}}{\mu m g}$ ), if $R_{1}$ and $R_{2}$ are the physical reactions of mass 1 and mass 2 , and $\varepsilon$ is the rescaled driving force applied on mass 1 .

Because of the nonsmooth Coulomb law, the unknowns are not only $u_{1}$ and $u_{2}$ but also $r_{1}$ and $r_{2}$, so that problem (4) is a Cauchy problem combined with a differential inclusion. The initial values of the friction forces correspond to the fact that, before adding the driving force, mass 1 is at rest but in imminent sliding to the right and mass 2 is also at rest but in imminent sliding to the left. This means that mass 1 starts moving as soon as any positive driving force is applied. Note that if the reaction of mass 1 were strictly inside the Coulomb cone, the reaction of mass 2 would also be strictly inside the Coulomb cone (at an equilibrium $r_{1}+r_{2}=0$ ) and therefore any perturbation smaller than $\bar{\varepsilon}=r_{1}(0)+1$ would fail to put mass 1 into motion. In such a case the conjecture would be trivially in agreement with experience.

The motion of mass 1 until it either stops or puts mass 2 in motion is governed by the following differential equation:

$\left\{\begin{array}{l}\ddot{u}_{1}+u_{1}=-1+\varepsilon, \quad t>0, \\ u_{1}(0)=-1, \quad \dot{u}_{1}(0)=0,\end{array}\right.$

so that:

$\left\{\begin{array}{l}u_{1}(t)=\varepsilon(1-\cos (t))-1, \\ \dot{u}_{1}(t)=\varepsilon \sin (t) .\end{array}\right.$

Mass 2 shall not move while $r_{2}(t)=-u_{1}(t)=1-$ $\varepsilon(1-\cos (t)) \geq-1$ and if $\varepsilon<1$, then $r_{2}(t)>-1 \forall t$. So that when mass 1 stops the reactions of both masses shall be strictly inside the Coulomb cone which gives a first case in agreement with the conjecture.

This result holds for a chain of any number of masses as long as the mass next to the one in immi- nent sliding on which the external force $\varepsilon$ is exerted, is strictly inside the Coulomb cone in the initial equilibrium state.

If two adjacent masses are in imminent sliding to the right, the rescaled system which corresponds to the motion of the two masses with external force $\varepsilon$ exerted on mass 1 and the reaction $\bar{r}_{3}$ of mass 3 strictly inside the Coulomb cone, is:

$\left\{\begin{array}{l}\ddot{u}_{1}+\left(u_{1}-u_{2}\right)=r_{1}+\varepsilon, \\ \ddot{u}_{2}+2 u_{2}-u_{1}-u_{3}=r_{2}, \quad t>0 \\ u_{3}=0 \\ -1 \leq r_{1} \leq+1, \quad-1 \leq r_{2} \leq+1, \\ -1 \leq r_{3} \leq+1, \\ u_{1}(0)=-3, \quad u_{2}(0)=-2, \quad u_{3}(0)=0 \\ \dot{u}_{1}(0)=\dot{u}_{2}(0)=0, \\ r_{1}(0)=-1, \quad r_{2}(0)=-1, \\ \left.r_{3}(0)=\bar{r}_{3} \in\right]-1,+1[.\end{array}\right.$

The motion of the two masses modifies the reaction of mass 3 and system (5) gives:

$$
\begin{aligned}
r_{3}(t)= & \bar{r}_{3}-\varepsilon\left(1+\frac{3-\sqrt{5}}{2 \sqrt{5}} \cos \left(\omega_{1} t\right)\right. \\
& \left.-\frac{3+\sqrt{5}}{2 \sqrt{5}} \cos \left(\omega_{2} t\right)\right),
\end{aligned}
$$

where $\omega_{1}^{2}=\frac{3+\sqrt{5}}{2}$ and $\omega_{2}^{2}=\frac{3-\sqrt{5}}{2}$.

So that once again for sufficiently small values of the external perturbation $\varepsilon$, we can be assured that $r_{3}(t)$ stays strictly greater than -1 ; in other words, that the reaction of mass 3 stays strictly inside the Coulomb cone. Therefore, when the first two masses stop moving the reactions of all three masses jump strictly inside the Coulomb cone. 


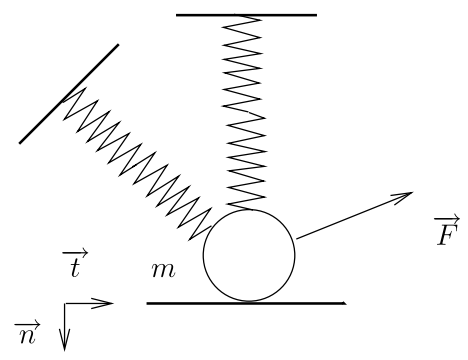

Fig. 2 Klarbring's model

This kind of result, providing in particular a value for a bound on the perturbation $\varepsilon$ which leads to an equilibrium solution strictly inside the Coulomb cone, can be easily extended to the case when any number of adjacent masses are simultaneously in imminent sliding on the same side of the cone in the initial configuration. Note that no more than half the number of masses can be concerned as the system is in an equilibrium state before the perturbation is applied (therefore the sum of all the reactions must be equal to zero).

\subsection{Klarbring's model}

We now consider the classical mass-spring system represented in Fig. 2.

Indices $n$ and $t$ will denote respectively, as in (3), the normal and tangential components of the displacement $u$ and of the reaction $R$. The mass shall be in unilateral contact with the horizontal plane and submitted to Coulomb friction. The motion of the mass shall be described by the following differential system:

$$
\left\{\begin{array}{l}
m \ddot{u}_{t}+K_{t} u_{t}+W u_{n}=F_{t}+R_{t}, \quad \\
m \ddot{u}_{n}+W u_{t}+K_{n} u_{n}=F_{n}+R_{n}, \\
u_{n} \leq 0, \quad R_{n} \leq 0, \quad u_{n} R_{n}=0, \\
\mu R_{n} \leq R_{t} \leq-\mu R_{n} .
\end{array} t>0\right.
$$

This system has been studied in [5] where it has been shown that the equilibrium solutions are all in strict contact when the quantity $A=K_{t} F_{n}-W F_{t}$ is strictly positive. If we consider an initial equilibrium where the reactions are strictly inside the Coulomb cone, i.e. a space equilibrium, then we can find $\varepsilon>0$ such that any perturbation of the external forces smaller than $\varepsilon$ shall leave the mass motionless, so that such a case is trivially in agreement with the conjecture. We observe in Fig. 3 that given $\left(R_{t}^{*}, R_{n}^{*}\right)$ strictly inside the cone,

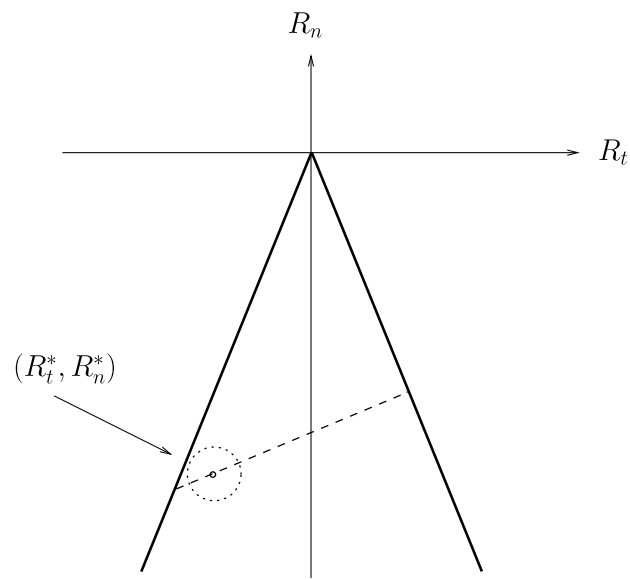

Fig. 3 A space equilibrium

a straightforward calculation gives the radius of a ball centered on $\left(R_{t}^{*}, R_{n}^{*}\right)$ and included in the cone.

We have therefore only to consider, among the equilibrium solutions that are strictly in contact, those which are in imminent sliding. When $K_{t}-\mu W>0$ there are two equilibrium solutions in imminent sliding (one to the right and one to the left), whereas when $K_{t}-\mu W \leq 0$ there is only one equilibrium solution in imminent sliding to the left (see [5]). The set of normal components of the reaction at time $t$ corresponding to a strictly stuck equilibrium solution when $K_{t}-\mu W>0$ is given by the following segment (corresponding to the projection on the $R_{n}$ axis of the dashed line of Fig. 3):

$$
\begin{aligned}
\left\{R_{n}\right\}(t)= & {\left[\frac{-K_{t} F_{n}(t)+W F_{t}(t)}{K_{t}-\mu W},\right.} \\
& \left.\frac{-K_{t} F_{n}(t)+W F_{t}(t)}{K_{t}+\mu W}\right],
\end{aligned}
$$

and when $K_{t}-\mu W \leq 0$ it is a half line given by:

$\left.\left.\left\{R_{n}\right\}(t)=\right]-\infty, \frac{-K_{t} F_{n}(t)+W F_{t}(t)}{K_{t}+\mu W}\right]$.

Before studying different types of perturbations we establish the following lemma which shall be a very useful technical tool.

Lemma 1 Let the loading be piecewise analytical and let $\left\{R_{n}\right\}(t)$ be the set of normal components of the reactions at time $t$ corresponding to a strictly stuck equilibrium solution. We suppose that $A>0$ and we con- 
sider the trajectory of a sliding mass which satisfies problem (6).

If at the instant $t^{*}$ when the mass stops sliding, its normal reaction $R_{n}^{*}$ belongs to the interior of $\left\{R_{n}\right\}\left(t^{*}\right)$, then the mass shall remain in a strictly stuck equilibrium state as long as its normal reaction belongs to the interior of $\left\{R_{n}\right\}(t)$.

Proof An existence and uniqueness result for problem (6) has been established in [4] when the loading is piecewise analytical. So that any continuous function whose first derivative is of bounded variation that satisfies all the relations in (6) shall be the unique solution of (6).

Let $\left(u_{t}(t), R_{t}(t), R_{n}(t)\right)$ be a solution of (6) in $\left[0, t^{*}\right]$ strictly in contact (i.e. $u_{n}(t)=0$ as $A>0$ ) such that $\dot{u}_{t}\left(t^{*}\right)=0$ and $R_{n}^{*}$ belongs to the interior of the set $\left\{R_{n}\right\}\left(t^{*}\right)$. Then we can extend this solution for $t^{*}<t \leq \bar{t}$ by:

$u_{t}(t)=u_{t}\left(t^{*}\right), \quad \dot{u}_{t}(t)=0$,

$R_{t}(t)=K_{t} u_{t}\left(t^{*}\right)-F_{t}(t) \quad$ and

$R_{n}(t)=W u_{t}\left(t^{*}\right)-F_{n}(t)$.

We define $\bar{t}$ as the first instant for which $R_{n}(t)$ does not belong to the set $\left\{R_{n}\right\}(t)$. If $R_{n}(t)$ belongs to the interior of $\left\{R_{n}\right\}(t)$ for all $t$, then the solution obtained is a strictly stuck equilibrium solution.

Remark When the loading $F$ is constant in time then Lemma 1 can be written in the following simplified and stronger terms:

If the mass stops at time $t^{*}$ for which the normal component of the reaction $R_{n}^{\star}$ is equal to the normal component of a reaction at equilibrium, then the particle remains at rest for all time $t>t^{*}$.

From now on we shall write the loadings $F_{t}(t)$ and $F_{n}(t)$ in the following way:

$F_{t}(t)=F_{t}+P_{t}(t)$ and $F_{n}(t)=F_{n}+P_{n}(t)$, where $P_{t}(t)$ and $P_{n}(t)$ are respectively a tangential perturbation and a normal one. We shall also for the sake of simplicity consider only tangential perturbations. It is easy to check that adding a normal perturbation yields the same results.

\subsubsection{A constant perturbation}

We begin by considering an equilibrium solution in imminent sliding to the right, in which case $K_{t}-$ $\mu F_{n}>0$ and $u_{t}=\frac{F_{t}-\mu F_{n}}{K_{t}-\mu W}, u_{n}=0, R_{n}=\frac{-A}{K_{t}-\mu W}$ with $R_{t}=\mu R_{n}$.

We then apply a constant tangential perturbation $\varepsilon$. In this case the set of normal components of the reaction corresponding to strictly stuck equilibrium solutions is time-independent and given by:

$\bar{R}_{n}=\left[\frac{-A+\varepsilon W}{K_{t}-\mu W}, \frac{-A+\varepsilon W}{K_{t}+\mu W}\right]$.

If the perturbation $\varepsilon$ is strictly negative then the reaction jumps to a value strictly inside the Coulomb cone and the mass is in a strictly stuck equilibrium state. On the other hand, if $\varepsilon$ is strictly positive then the mass starts sliding to the right and its motion satisfies the following differential equation:

$\left\{\begin{array}{l}m \ddot{u}_{t}+\left(K_{t}-\mu W\right) u_{t}=F_{t}+\varepsilon-\mu F_{n}, \\ u_{t}(0)=\frac{F_{t}-\mu F_{n}}{K_{t}-\mu W}, \quad \dot{u}_{t}(0)=0,\end{array} \quad t>0\right.$.

The solution of this equation is given by:

$u_{t}(t)=\frac{F_{t}-\mu F_{n}}{K_{t}-\mu W}+\frac{\varepsilon}{K_{t}-\mu W}(1-\cos (\alpha t))$,

where $\alpha$ is an intrinsic period of the sliding on the left side of the cone (sliding to the right for the mass) given by $\alpha^{2}=\frac{K_{t}-\mu W}{m}$. When the mass stops sliding at $t^{\star}=\frac{\pi}{\alpha}$, we have

$u_{t}\left(t^{\star}\right)=\frac{F_{t}-\mu F_{n}}{K_{t}-\mu W}+\frac{2 \varepsilon}{K_{t}-\mu W}$,

and

$R_{n}^{\star}=\frac{-A+2 \varepsilon W}{K_{t}-\mu W}$.

It is immediately seen that if $\varepsilon<\frac{2 \mu A}{K_{t}+3 \mu W}$, then

$\left.R_{n}^{\star} \in\right] \frac{-A+\varepsilon W}{K_{t}-\mu W}, \frac{-A+\varepsilon W}{K_{t}+\mu W}[$.

In other words, when the mass stops sliding there is a jump in the tangential reaction and for sufficiently small values of the perturbation the trajectory leads to an equilibrium state strictly inside the Coulomb cone.

A similar computation establishes that if we consider the equilibrium state which is in imminent sliding to the left, any positive tangential perturbation 
shall make the tangential reaction jump strictly inside the Coulomb cone, so that the mass shall be in a strictly stuck equilibrium state. Whereas a negative tangential perturbation shall make the mass move but when it stops sliding the jump in the tangential reaction shall bring the reaction strictly inside the Coulomb cone if $|\varepsilon|\left(K_{t}-3 \mu W\right)<2 \mu A$ when $K_{t}-$ $\mu W>0$ and for all values of $\varepsilon$ when $K_{t}-\mu W \leq 0$.

\subsubsection{A piecewise constant oscillating perturbation}

We consider a periodic perturbation of period $2 T$ equal to $\varepsilon$ on $] 2 i T,(2 i+1) T]$ and to 0 on $](2 i+$ 1) $T,(2 i+2) T]$, for $i=0, \ldots$. And we adopt the following notations:

$R_{n}^{-}=\frac{-A}{K_{t}-\mu W}$ for the reaction corresponding to imminent sliding to the right when no perturbation is added, and $R_{n}^{+}=\frac{-A}{K_{t}+\mu W}$ for the reaction corresponding to imminent sliding to the left;

$R_{n \epsilon}^{-}=\frac{-A+\varepsilon W}{K_{t}-\mu W}$ for the reaction corresponding to imminent sliding to the right when a constant perturbation $\varepsilon$ is added, and $R_{n \epsilon}^{+}=\frac{-A+\varepsilon W}{K_{t}+\mu W}$ for the reaction corresponding to imminent sliding to the left (see Fig. 4).

Note that if $\varepsilon>\frac{2 \mu A}{K_{t}+\mu W}$ then there is no equilibrium solution for such a perturbation. Indeed an equilibrium solution must be such that its normal reaction is both greater than $\frac{-A+\varepsilon W}{K_{t}-\mu W}$ and lower than $\frac{-A}{K_{t}+\mu W}$ (see Fig. 4). So that to ensure that the set of possible values for the normal reaction is not empty (i.e. that $R_{n \varepsilon}^{-}<R_{n}^{+}$) we must choose $\varepsilon$ smaller than $\frac{2 \mu A}{K_{t}+\mu W}$.
The solution in the first time interval ]0, T], for such an oscillating perturbation, is equal to:

$u_{t}(t)=\frac{F_{t}-\mu F_{n}}{K_{t}-\mu W}+\frac{\varepsilon}{K_{t}-\mu W}(1-\cos (\alpha t))$,

and we now continue the discussion with respect to the half period $T$.

If $T \geq \frac{\pi}{\alpha}$ then the mass stops at $t^{\star}=\frac{\pi}{\alpha}$. If $T>\frac{\pi}{\alpha}$ there is a jump in the tangential reaction and if the perturbation is sufficiently small, namely $\varepsilon<\frac{\mu A}{K_{t}+\mu W}$, a strictly stuck equilibrium solution shall be obtained.

If $T=\frac{\pi}{\alpha}$ then, when the mass stops, its reactions shall be on the edge of the Coulomb cone in imminent sliding as long as $\varepsilon<\frac{\mu A}{K_{t}+\mu W}$.

If on the other hand $T<\frac{\pi}{\alpha}$, then there exists an integer $n$ such that

$\frac{\pi}{(2 n+1) \alpha} \leq T<\frac{\pi}{(2 n-1) \alpha}$

and the solution of the perturbed system shall be given by:

For $i=0, \ldots, n-1$,

$$
\left\{\begin{aligned}
\text { For }(2 i) & T<t \leq(2 i+1) T \\
u_{t}(t)= & \frac{F_{t}-\mu F_{n}}{K_{t}-\mu W} \\
& +\frac{\varepsilon}{K_{t}-\mu W}\left(1-\sum_{j=0}^{2 i}(-1)^{j} \cos (\alpha(t-j T)),\right. \\
= & \frac{F_{t}-\mu F_{n}}{K_{t}-\mu W}+\frac{\varepsilon}{K_{t}-\mu W}\left(1-(-1)^{i} \cos (\alpha(t-i T))\right. \\
& \left.\times\left(1+2 \sum_{j=1}^{i}(-1)^{j} \cos (j \alpha T)\right)\right),
\end{aligned}\right.
$$

Fig. 4 The Coulomb cone (dashed line: non-perturbed equilibrium solutions; dotted line: perturbed equilibrium solutions)

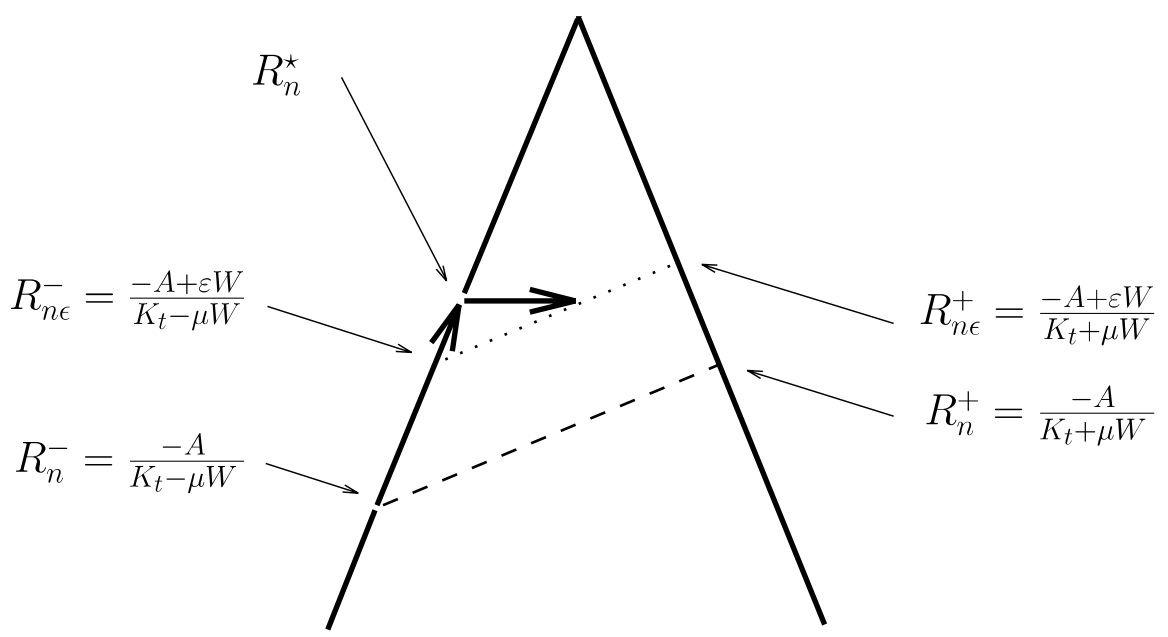




$$
\left\{\begin{aligned}
\text { For }(2 i & +1) T<t \leq(2 i+2) T \\
u_{t}(t)= & \frac{F_{t}-\mu F_{n}}{K_{t}-\mu W} \\
& -\frac{\varepsilon}{K_{t}-\mu W} \sum_{j=0}^{2 i+1}(-1)^{j} \cos (\alpha(t-j T)) \\
= & \frac{F_{t}-\mu F_{n}}{K_{t}-\mu W}+\frac{2 \varepsilon}{K_{t}-\mu W} \sin \frac{\alpha(2 t-(2 i+1) T)}{2} \\
& \times \sum_{j=0}^{i}(-1)^{i-j} \sin \left(\frac{(2 j+1) \alpha T}{2}\right) .
\end{aligned}\right.
$$

The mass slides to the right and cannot stop until it has reached the time interval $](2 n-1) T, 2 n T]$. As a matter of fact the derivative of the solution cannot be equal to zero in the intervals $] 2 i T,(2 i+1) T]$ because $\dot{u}_{t}\left(\tilde{t}_{i}\right)=0$ in such an interval would imply $\tilde{t}_{i}=\frac{\pi}{\alpha}+i T$ and

$\tilde{t}_{i}>(2 n-1) T+i T \geq(2 i+1) T$ for $i=0, \ldots, n-1$.

In the same way the sliding velocity cannot be equal to zero in the intervals $](2 i+1) T,(2 i+2) T]$ either, because $\dot{u}_{t}\left(\tilde{t}_{i}\right)=0$ implies that $\tilde{t}_{i}=\frac{\pi}{2 \alpha}+(2 i+1) \frac{T}{2}$, but as $\frac{\pi}{\alpha}>(2 n-1) T$, we have

$\tilde{t}_{i}>(2 i+2) T$ as long as $i \leq n-2$,

and $\tilde{t}_{i}$ does not belong to $\left.](2 i+1) T,(2 i+2) T\right]$.

However, in the time interval $](2 n-1) T, 2 n T]$ the derivative of the solution is equal to zero for $\tilde{t}=\frac{\pi}{2 \alpha}+$ $(2 n-1) \frac{T}{2}$ which belongs to $\left.](2 n-1) T, 2 n T\right]$ since $\frac{\pi}{(2 n+1) \alpha} \leq T<\frac{\pi}{(2 n-1) \alpha}$.

When the mass comes to a rest at $\tilde{t}$, a jump in the tangential reaction shall bring the reactions strictly inside the Coulomb cone. To ensure that we have obtained a space equilibrium we must check that the reaction stays strictly inside the cone for all future time, in particular when the perturbation $\varepsilon$ is applied once more. We must therefore compute the normal reaction $R_{n}(\tilde{t})$ at $\tilde{t}$ and make sure that $R_{n \varepsilon}^{-} \leq R_{n}(\tilde{t}) \leq R_{n}^{+}$. We have:

$R_{n}(\tilde{t})=\frac{-A+2 \varepsilon W\left[\sum_{j=0}^{n-1}(-1)^{n-1-j} \sin \frac{(2 j+1) \alpha T}{2}\right]}{K_{t}-\mu W}$.

So that we must check that

$$
R_{n}(\tilde{t}) \geq R_{n \varepsilon}^{-}=\frac{-A+\varepsilon W}{K_{t}-\mu W}
$$

$$
\text { when } \frac{\pi}{(2 n+1) \alpha} \leq T<\frac{\pi}{(2 n-1) \alpha} \text {. }
$$

By writing

$$
\begin{aligned}
& \sum_{j=0}^{n-1}(-1)^{n-1-j} \sin \left(\frac{(2 j+1) \alpha T}{2}\right) \\
& \quad=(-1)^{n-1} \sum_{j=0}^{n-1}(-1)^{j} \Im\left(e^{i(2 j+1) \frac{\alpha T}{2}}\right)
\end{aligned}
$$

and

$$
\begin{aligned}
& \sum_{j=0}^{n-1}(-1)^{n-1-j} \sin \left(\frac{(2 j+1) \alpha T}{2}\right) \\
& \quad=(-1)^{n-1} \Im\left(e^{i \frac{\alpha T}{2}} \sum_{j=0}^{n-1}\left(-e^{i \alpha T}\right)^{j}\right),
\end{aligned}
$$

we obtain that

$$
\sum_{j=0}^{n-1}(-1)^{n-1-j} \sin \left(\frac{(2 j+1) \alpha T}{2}\right)=\frac{\sin (n \alpha T)}{2 \cos \left(\frac{\alpha T}{2}\right)} .
$$

The roots of

$f(T)=\sin (n \alpha T)-\cos \left(\frac{\alpha T}{2}\right)$

are equal to

$\frac{\pi}{(2 n+1) \alpha}+\frac{4 k \pi}{(2 n+1) \alpha}$ and

$\frac{\pi}{(2 n-1) \alpha}+\frac{4 k \pi}{(2 n-1) \alpha}$ for $k \in \mathbb{Z}$.

So that we have for both

$$
\begin{aligned}
& T=\frac{\pi}{(2 n+1) \alpha} \quad \text { and } \quad T=\frac{\pi}{(2 n-1) \alpha}, \\
& \frac{\sin (n \alpha T)}{\cos \left(\frac{\alpha T}{2}\right)}=1,
\end{aligned}
$$

and for all other values of $T$ in $] \frac{\pi}{(2 n+1) \alpha}, \frac{\pi}{(2 n-1) \alpha}[$, $\frac{\sin (n \alpha T)}{\cos \left(\frac{\alpha T}{2}\right)}>1$.

This enables us to state that:

$R_{n}(\tilde{t})=\frac{-A+2 \varepsilon W \frac{\sin (n \alpha T)}{2 \cos \left(\frac{\alpha T}{2}\right)}}{K_{t}-\mu W} \geq \frac{-A+\varepsilon W}{K_{t}-\mu W}=R_{n \varepsilon}^{-}$. 
Fig. 5 Space equilibria for the sinusoidal perturbation: reactions corresponding to non-perturbed equilibrium solutions are on the dashed line; reactions corresponding to perturbed equilibrium solutions are between the two dotted lines

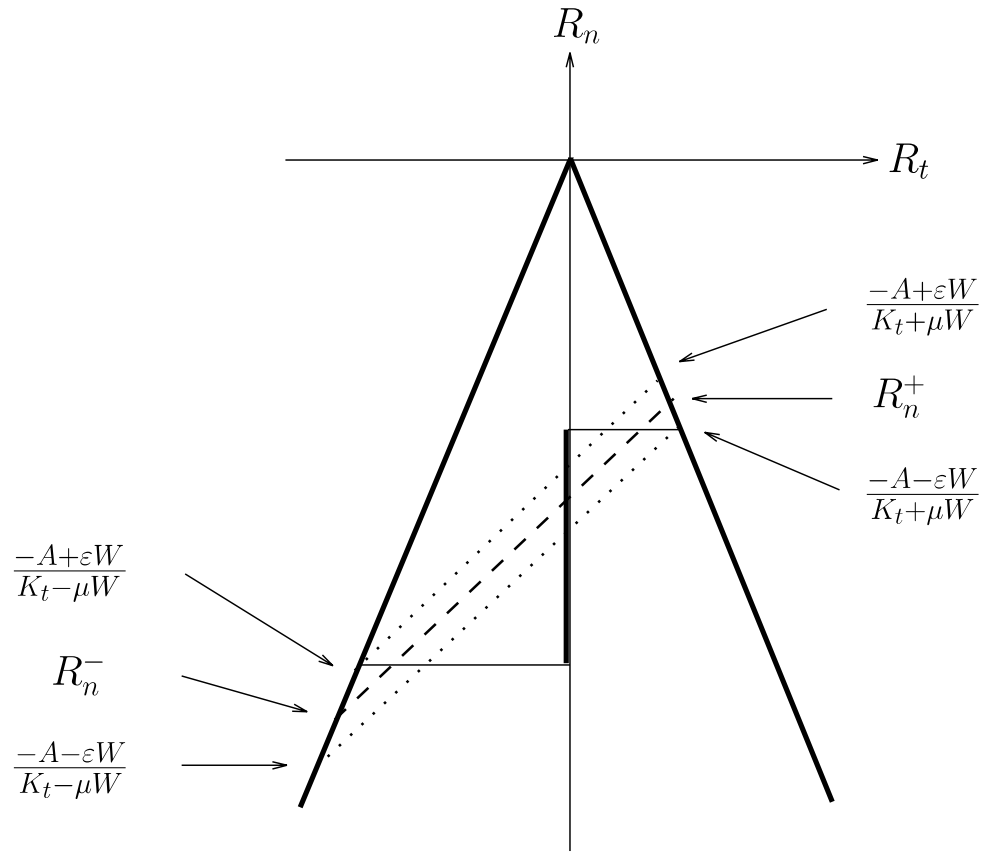

But the normal reaction $R_{n}(\tilde{t})$ must also be lower than $\frac{-A}{K_{t}+\mu W}$.

As we always have $\frac{\sin (n \alpha T)}{\cos \left(\frac{\alpha T}{2}\right)} \leq 2$, we have that $R_{n}(\tilde{t}) \leq \frac{-A+2 \varepsilon W}{K_{t}-\mu W}$, so that if $\varepsilon<\frac{\mu A}{K_{t}+\mu W}$ then $R_{n}(\tilde{t})$ shall be lower than $R_{n}^{+}=\frac{-A}{K_{t}+\mu W}$.

We have shown that if the amplitude of the periodic perturbation $\varepsilon$ is sufficiently small then when the mass stops sliding it reaches a strictly stuck equilibrium state whatever the frequency of the perturbation except when $T=\frac{\pi}{(2 n-1) \alpha}$, in which case the mass having started from an imminent sliding equilibrium stops in another imminent sliding equilibrium.

\subsubsection{A sinusoidal perturbation}

We now assume that a sinusoidal perturbation is added to the external loading, i.e. $P(t)=\varepsilon \sin (\gamma t)$. Then the bounds on the normal reaction also have a sinusoidal variation. Let us consider for the lower bound the maximum of $R_{n \varepsilon}^{+}(t)$ (obtained when $\sin (\gamma t)=1$ ), that is $\frac{-A+\varepsilon W}{K_{t}-\mu W}$, and for the upper bound the minimum of $R_{n \varepsilon}^{-}(t)$ (obtained when $\sin (\gamma t)=-1$ ), that is $\frac{-A-\varepsilon W}{K_{t}+\mu W}$, and assume there exists a time for which the mass stops with a normal reaction strictly inside the interval $] \frac{-A+\varepsilon W}{K_{t}-\mu W}, \frac{-A-\varepsilon W}{K_{t}+\mu W}[$; then it stays motionless for all future time. Due to Lemma 1 this condition on the normal component of the reaction when the mass stops furnishes a sufficient condition for the trajectory to lead to a space equilibrium.

We therefore choose $\varepsilon$ small enough (smaller than $\left.\frac{\mu A}{K_{t}}\right)$ to ensure that the interval $] \frac{-A+\varepsilon W}{K_{t}-\mu W}, \frac{-A-\varepsilon W}{K_{t}+\mu W}$ [ is not empty. This interval is represented by a thick line on the $R_{n}$ axis in Fig. 5. Let us first assume that $\gamma \neq \alpha$. The solution after applying the perturbation $P$ is:

$$
\begin{aligned}
u_{t}(t)= & \frac{F_{t}-\mu F_{n}}{K_{t}-\mu W} \\
& +\frac{\varepsilon}{\alpha m\left(\alpha^{2}-\gamma^{2}\right)}(\alpha \sin (\gamma t)-\gamma \sin (\alpha t)),
\end{aligned}
$$

until the mass stops sliding at $t^{*}=\frac{2 \pi}{\alpha+\gamma}$ and at that point the normal reaction shall be equal to

$R_{n}^{*}=\frac{-A}{K_{t}-\mu W}+\frac{\varepsilon W}{K_{t}-\mu W} \frac{\alpha}{\alpha-\gamma} \sin \left(\frac{\gamma 2 \pi}{\alpha+\gamma}\right)$.

And the motion shall cease if

$\frac{-A+\varepsilon W}{K_{t}-\mu W}<R_{n}^{*}<\frac{-A-\varepsilon W}{K_{t}+\mu W}$.

A simple computation shows that if $\gamma \in[0.18 \alpha, 1.76 \alpha]$ then $\frac{-A+\varepsilon W}{K_{t}-\mu W}<R_{n}^{*}$.

When $\gamma=\alpha$, the solution is given by:

$u_{t}(t)=\frac{F_{t}-\mu F_{n}}{K_{t}-\mu W}-\frac{\varepsilon \alpha t \cos (\alpha t)}{2\left(K_{t}-\mu W\right)}+\frac{\varepsilon \sin (\alpha t)}{2\left(K_{t}-\mu W\right)}$, 
and this time when the mass stops sliding its normal reaction is equal to

$R_{n}^{*}=\frac{-A+\varepsilon W \pi / 2}{K_{t}-\mu W}$.

So that all values of the frequency of the perturbing force in $[0.18 \alpha, 1.76 \alpha]$ (including $\gamma=\alpha$ ) lead to a stop at $t^{*}$ where the reaction is such that:

$R_{n}^{*}>\frac{-A+\varepsilon W}{K_{t}-\mu W}$.

Let us now assume that condition (10) is satisfied.

If

$$
\varepsilon<\frac{2 A \mu}{K_{t}-\mu W+\left(K_{t}+\mu W\right) \frac{\alpha}{\alpha-\gamma} \sin \left(\frac{\gamma 2 \pi}{\alpha+\gamma}\right)},
$$

when $\alpha \neq \gamma$,

and

$\varepsilon<\frac{2 A \mu}{K_{t}-\mu W+\left(K_{t}+\mu W\right) \frac{\pi}{2}}, \quad$ when $\alpha=\gamma$,

then:

$R_{n}^{*}<\frac{-A-\varepsilon W}{K_{t}+\mu W}$.

We have therefore shown that when a periodic perturbation is applied for certain values of the frequency of the perturbation the mass stops after just one phase of sliding to the right and then that, if $\varepsilon$ is sufficiently small, it shall stay motionless. For all the other values of the frequency of the perturbation the mass either has a certain number of sliding phases to the right before stoping (as in the case of small periods of piecewise constant oscillating perturbations) or slides alternatively to the right and to the left before stoping for sufficiently small values of $\varepsilon$. This can be checked for instance by computing the solution through a Maple software.

\section{A slightly more complicated mass-spring system}

The problem we consider is represented in Fig. 6, where $\phi$ is the angle between the springs, the two bodies are of mass $m$ and the stiffness of the springs is equal to $k$. In the following we shall denote by $c$ the data $\cos \phi$ and by $s$ the data $\sin \phi$.

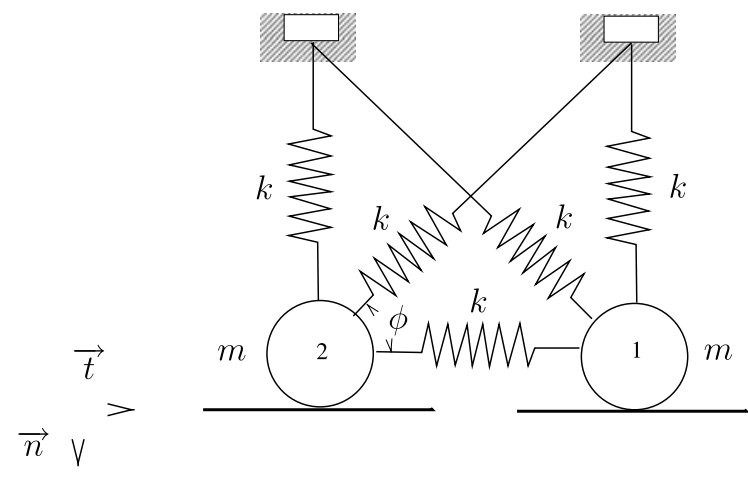

Fig. 6 The two-mass problem

This simple system is a generalization of Klarbring's model [9], seen in the preceding section, and was first studied in [1] and more recently in [12]. The movement of the two masses is governed by (11)-(15). In (11) below, the parameters $k$ and $m$ have been taken equal to 1 (an adequate rescaling would have had the same effect).

- The equations:

$\left\{\begin{array}{l}\ddot{u}_{1 t}+\left(1+c^{2}\right) u_{1 t}+c s u_{1 n}-u_{2 t}=F_{1 t}+R_{1 t}, \\ \ddot{u}_{1 n}+c s u_{1 t}+\left(1+s^{2}\right) u_{1 n}=F_{1 n}+R_{1 n}, \\ \ddot{u}_{2 t}-u_{1 t}+\left(1+c^{2}\right) u_{2 t}-c s u_{2 n}=F_{2 t}+R_{2 t}, \\ \ddot{u}_{2 n}-c s u_{2 t}+\left(1+s^{2}\right) u_{2 n}=F_{2 n}+R_{2 n} .\end{array}\right.$

- The initial conditions:

For $i=1,2$

$$
\begin{array}{ll}
u_{i t}(0)=u_{i t 0}, & \dot{u}_{i t}(0)=v_{i t 0}, \\
u_{i n}(0)=u_{i n 0}, & \dot{u}_{i n}(0)=v_{i n} 0 .
\end{array}
$$

- The unilateral contact conditions:

For $i=1,2$

$$
R_{\text {in }} \leq 0, \quad u_{\text {in }} \leq 0, \quad R_{\text {in }} u_{\text {in }}=0 .
$$

- The Coulomb friction law:

For $i=1,2 \quad\left|R_{i t}\right| \leq-\mu R_{i n} \quad$ and

$\left\{\begin{aligned}\left|R_{i t}\right| & <-\mu R_{i n} \Longrightarrow \dot{u}=0, \\ \left|R_{i t}\right| & =-\mu R_{i n} \Longrightarrow \\ \exists \lambda & \geq 0 \text { such that } \dot{u}=-\lambda R_{i t} .\end{aligned}\right.$ 
- The impact law:

For $i=1,2 \quad$ when $u_{\text {in }}(t)=0$,

$\dot{u}_{i n}\left(t^{+}\right)=-e \dot{u}_{i n}\left(t^{-}\right) \quad$ with $e \in[0,1]$.

In system (11)-(15), $R_{i t}$ and $R_{i n}, i=1,2$, are respectively the tangential and the normal components of the reaction exerted by the obstacle on mass 1 and mass $2 ; \mu$ is the friction coefficient; $u_{i t}$ and $u_{i n}$ the tangential and the normal components of the displacement, and (.) stands for the time derivative. $F_{i t}$ and $F_{i n}$ are the tangential and normal components of the external loading. The initial conditions (12) are supposed compatible with the unilateral conditions (13). The impact law (15) can be expressed in this way as soon as $\dot{u}_{i n}$ is of bounded variation, which in addition to contact conditions (13) implies that $\ddot{u}$ and $R$ in (11) are measures (see e.g. [4, 11]).

\subsection{Equilibrium states}

We begin by determining all the equilibrium solutions of problem (11)-(15) in the case where the external forces are constant. These equilibrium solutions must satisfy the following relations:

$\left\{\begin{array}{l}\left(1+c^{2}\right) u_{1 t}+c s u_{1 n}-u_{2 t}=F_{1 t}+R_{1 t}, \\ \operatorname{csu}_{1 t}+\left(1+s^{2}\right) u_{1 n}=F_{1 n}+R_{1 n}, \\ -u_{1 t}+\left(1+c^{2}\right) u_{2 t}-c s u_{2 n}=F_{2 t}+R_{2 t}, \\ -\operatorname{csu}_{2 t}+\left(1+s^{2}\right) u_{2 n}=F_{2 n}+R_{2 n} .\end{array}\right.$

For $i=1,2 \quad R_{\text {in }} \leq 0, \quad u_{\text {in }} \leq 0, \quad R_{\text {in }} u_{\text {in }}=0$.

For $i=1,2 \quad\left|R_{i t}\right| \leq-\mu R_{i n}$.

An equilibrium solution is given by the set $\left(u_{i n}, u_{i t}\right.$, $R_{i n}, R_{i t}$ ) for $i=1,2$ that satisfies (16), (17) and (18). Due to conditions (17) and (18) these relations define a strongly nonlinear system; however, we observe that looking for a solution with no contact amounts to inserting into system (16) the $R_{i n}=0, i=1,2$, and the displacements must satisfy (17), whereas looking for a solution in contact amounts to inserting into system (16) the $u_{\text {in }}=0, i=1,2$, but in this case the reactions must satisfy (17) and (18).

We generalize here the one-mass case studied in [5]. However, in [5] it was relatively easy to describe extensively the different equilibrium states according to the different values of the parameters and these equilibrium states could be summarized in a table. Here such a table would be much too complicated so that we shall simply describe the different types of equilibrium states and give the values of the parameters that lead to such states.

\subsubsection{The two masses are not in contact}

The displacements of the equilibrium solution corresponding to the no contact case are obtained by solving the linear system (16) with $R_{i n}=0, i=1,2$. However, this shall correspond to an equilibrium solution only if conditions (17) are fulfilled. As the reactions are zero, these conditions are reduced to $u_{i n} \leq 0, i=1,2$. The normal components of the displacements are given by:

$u_{1 n}=\frac{\AA_{1}}{4 c^{2}-c^{4}} \quad$ and $\quad u_{2 n}=\frac{\AA_{2}}{4 c^{2}-c^{4}}$

where quantities $\AA_{1}$ and $\AA_{2}$ depend only on the data and are defined by:

$$
\begin{aligned}
\AA_{1}= & 3 c^{2} F_{1 n}-s^{2} c^{2} F_{2 n} \\
& -2 s c F_{1 t}-s c\left(1+s^{2}\right) F_{2 t}, \\
\AA_{2}= & 3 c^{2} F_{2 n}-s^{2} c^{2} F_{1 n} \\
& +2 s c F_{2 t}+s c\left(1+s^{2}\right) F_{1 t} .
\end{aligned}
$$

As $4 c^{2}-c^{4}>0$, the solution of (16) thus obtained is an equilibrium solution only if $\AA_{1} \leq 0$ and $\AA_{2} \leq 0$. If either $\AA_{1}>0$ or $\AA_{2}>0$, there is no equilibrium solution where the two masses are not in contact.

\subsubsection{Only one mass is in contact}

We now determine the equilibrium solution which corresponds to one mass in contact and the other not. We assume that mass 2 is in contact and mass 1 is not. We thus have to solve the following problem:

$\left\{\begin{array}{l}\left(1+c^{2}\right) u_{1 t}+c s u_{1 n}-u_{2 t}=F_{1 t}, \\ \operatorname{csu}_{1 t}+\left(1+s^{2}\right) u_{1 n}=F_{1 n}, \\ -u_{1 t}+\left(1+c^{2}\right) u_{2 t}=F_{2 t}+R_{2 t}, \\ -\operatorname{csu}_{2 t}=F_{2 n}+R_{2 n},\end{array}\right.$ 
Fig. 7 Equilibrium reactions in the $R_{2 t}, R_{2 n}$ plane; left: $d_{1} \leq 0$; right: $d_{1}>0$
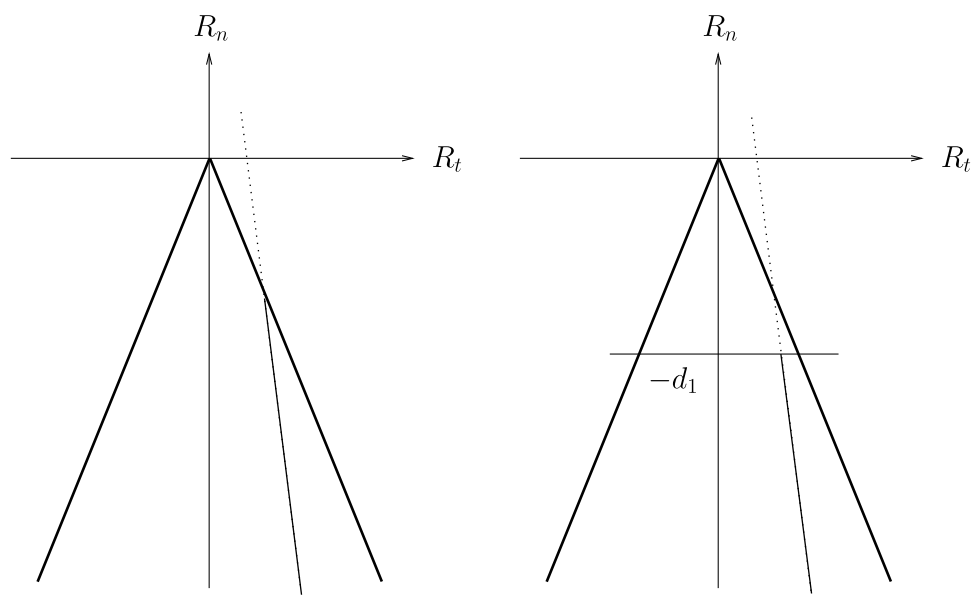

together with

$u_{1 n} \leq 0, \quad u_{2 n}=0$,

$R_{1 t}=R_{1 n}=0$,

$R_{2 n} \leq 0, \quad\left|R_{2 t}\right| \leq-\mu R_{2 n}$.

By expressing $u_{1 t}$ and $u_{2 t}$ in terms of the data and of $R_{2 t}$ and $R_{2 n}$, then eliminating $u_{1 n}$ one obtains the following relationship between the normal and tangential components of the reaction of mass 2 :

$$
\begin{aligned}
F_{1 t}- & \frac{F_{2 n}+R_{2 n}}{c s}+\left(1+c^{2}\right)\left[F_{2 t}+R_{2 t}\right. \\
& \left.+\frac{1+c^{2}}{c s}\left(F_{2 n}+R_{2 n}\right)\right] \\
= & \frac{c s}{1+s^{2}}\left[F_{1 n}+c s\left(F_{2 t}+R_{2 t}\right)\right. \\
& \left.+\left(1+c^{2}\right)\left(F_{2 n}+R_{2 n}\right)\right] .
\end{aligned}
$$

Introducing into the above expression $A_{2}$ defined in (19), we obtain:

$$
R_{2 n}=-\frac{2 s}{3 c} R_{2 t}-\frac{\AA_{2}}{3 c^{2}} .
$$

If $\AA_{2}<0$ and $\mu \leq \frac{3 c}{2 s}$, no equilibrium solution exists because no pair $\left(R_{2 t}, R_{2 n}\right)$ satisfies both the above relation and condition (21).

If $\AA_{2}<0$ and $\mu>\frac{3 c}{2 s}$, then any pair $\left(R_{2 t}, R_{2 n}\right)$ satisfying

$R_{2 n} \leq \frac{\AA_{2}}{c(2 s \mu-3 c)} \quad$ and $\quad R_{2 t}=-\frac{3 c}{2 s} R_{2 n}-\frac{\AA_{2}}{3 c s}$ corresponds to an equilibrium solution as long as $u_{1 n} \leq 0$. And $u_{1 n}$, the normal displacement of mass 1 , is given by:

$$
\begin{aligned}
u_{1 n}= & \frac{1}{1+s^{2}}\left[\left(1-\frac{c^{2}}{2}\right) R_{2 n}-\frac{\AA_{2}}{2}\right. \\
& \left.+F_{1 n}+c s F_{2 t}+\left(1+c^{2}\right) F_{2 n}\right] .
\end{aligned}
$$

We introduce at this point two quantities that depend uniquely on the data:

$d_{1} \stackrel{\text { def }}{\equiv}\left(1+c^{2}\right) F_{1 n}+F_{2 n}-c s F_{1 t}$,

$d_{2} \stackrel{\text { def }}{\equiv} F_{1 n}+\left(1+c^{2}\right) F_{2 n}+c s F_{2 t}$.

It is easily seen that quantities $d_{1}$ and $d_{2}$ are related to $\AA_{1}$ and $\AA_{2}$ in the following way:

$\AA_{1}=2 d_{1}+\left(c^{2}-2\right) d_{2}$,

$\AA_{2}=\left(c^{2}-2\right) d_{1}+2 d_{2}$.

So that finally the normal displacement of mass 1 can be expressed as:

$u_{1 n}=\frac{R_{2 n}+d_{1}}{2}$.

We see by (25) that if $d_{1} \leq 0$ then all the pairs $\left(R_{2 t}, R_{2 n}\right)$ defined above are compatible with the unilateral conditions, whereas if $d_{1}>0$ then only the pairs such that $R_{2 n} \leq-d_{1}$ give rise to an equilibrium solution. Figure 7 represents in the $\left(R_{2 t}, R_{2 n}\right)$ plane the sets of $\left(R_{2 t}, R_{2 n}\right)$ corresponding to an equilibrium solution when $d_{1} \leq 0$ and when $d_{1}>0$. We obtain the 
same figure as in the one-mass case (cf. [5]): when $d_{1} \leq 0$ but when $d_{1}>0$, the pairs of $\left(R_{2 t}, R_{2 n}\right)$ corresponding to an equilibrium solution must also satisfy $R_{2 n} \leq-d_{1}$ because of (21) and (25).

We have given here the results obtained when looking for an equilibrium solution where mass 1 is not in contact and mass 2 is. The results obtained when mass 2 is not in contact and mass 1 is, are given by similar expressions where the subscripts 1 and 2 have been interchanged.

\subsubsection{The two masses are in contact}

When the two masses are in contact, both $u_{1 n}$ and $u_{2 n}$ are equal to zero so that the equilibrium solution is obtained by solving the following problem:

$\left\{\begin{array}{l}\left(1+c^{2}\right) u_{1 t}-u_{2 t}=F_{1 t}+R_{1 t}, \\ \operatorname{cs}_{1 t}=F_{1 n}+R_{1 n}, \\ -u_{1 t}+\left(1+c^{2}\right) u_{2 t}=F_{2 t}+R_{2 t}, \\ -\operatorname{csu}_{2 t}=F_{2 n}+R_{2 n},\end{array}\right.$

with:

$R_{1 n} \leq 0, \quad R_{2 n} \leq 0$,

$u_{1 n}=0, \quad u_{2 n}=0$,

$\left|R_{1 t}\right| \leq \mu R_{1 n}, \quad\left|R_{2 t}\right| \leq \mu R_{2 n}$.

Eliminating $u_{1 t}$ and $u_{2 t}$, we have:

$\left(1+c^{2}\right)\left(F_{1 n}+R_{1 n}\right)+F_{2 n}+R_{2 n}=c s\left(F_{1 t}+R_{1 t}\right)$,

$-\left(F_{1 n}+R_{1 n}\right)-\left(1+c^{2}\right)\left(F_{2 n}+R_{2 n}\right)$

$$
=c s\left(F_{2 t}+R_{2 t}\right) \text {, }
$$

which we can rewrite as:

$$
\begin{aligned}
& \left(1+c^{2}\right) R_{1 n}+R_{2 n}-c s R_{1 t} \\
& \quad=-\left[\left(1+c^{2}\right) F_{1 n}+F_{2 n}-c s F_{1 t}\right], \\
& R_{1 n}+\left(1+c^{2}\right) R_{2 n}+c s R_{2 t} \\
& \quad=-\left[F_{1 n}+\left(1+c^{2}\right) F_{2 n}+c s F_{2 t}\right] .
\end{aligned}
$$

By introducing into the above expressions $d_{1}$ and $d_{2}$ defined by (23) we have that the following relations must be satisfied by $R_{1 n}, R_{2 n}, R_{1 t}$ and $R_{2 t}$ :

$\left\{\begin{array}{l}\left(1+c^{2}\right) R_{1 n}+R_{2 n}-c s R_{1 t}=-d_{1}, \\ R_{1 n}+\left(1+c^{2}\right) R_{2 n}+c s R_{2 t}=-d_{2} .\end{array}\right.$
The vector $u=\left(R_{1 t}, R_{1 n}, R_{2 t}, R_{2 n}\right)$ belongs to $\mathbb{R}^{4}$ and the vectors $u$ satisfying the above relations determine a plane $(\mathcal{P})$ in $\mathbb{R}^{4}$ defined by:

$$
\begin{aligned}
u \in(\mathcal{P}) \Longleftrightarrow u= & \frac{1}{c s}\left(\begin{array}{c}
d_{1} \\
0 \\
-d_{2} \\
0
\end{array}\right)+\frac{\alpha}{c s}\left(\begin{array}{c}
1+c^{2} \\
c s \\
-1 \\
0
\end{array}\right) \\
& +\frac{\beta}{c s}\left(\begin{array}{c}
1 \\
0 \\
-\left(1+c^{2}\right) \\
c s
\end{array}\right)
\end{aligned}
$$

with $(\alpha, \beta) \in \mathbb{R}^{2}$.

We can now express the relations (27) in the $(\alpha, \beta)$ plane in the following way:

$$
\begin{aligned}
R_{1 n} & \leq 0 \quad \Longleftrightarrow \quad \alpha \leq 0, \\
R_{2 n} & \leq 0 \quad \Longleftrightarrow \quad \beta \leq 0, \\
R_{1 t} & \leq-\mu R_{1 n} \\
& \Longleftrightarrow d_{1}+\alpha\left[\left(1+c^{2}\right)+\mu c s\right]+\beta \leq 0, \\
R_{1 t} & \geq \mu R_{1 n} \\
& \Longleftrightarrow d_{1}+\alpha\left[\left(1+c^{2}\right)-\mu c s\right]+\beta \geq 0,
\end{aligned}
$$

$R_{2 t} \leq-\mu R_{2 n}$

$$
\Longleftrightarrow d_{2}+\beta\left[\left(1+c^{2}\right)-\mu c s\right]+\alpha \geq 0,
$$

$R_{2 t} \geq \mu R_{2 n}$

$$
\Longleftrightarrow d_{2}+\beta\left[\left(1+c^{2}\right)+\mu c s\right]+\alpha \leq 0 .
$$

We introduce the following notations that we shall adopt from now on:

$\mathcal{C} \stackrel{\text { def }}{\equiv}\left(1+c^{2}\right)+\mu c s, \quad \mathcal{C}^{\prime} \stackrel{\text { def }}{\equiv}\left(1+c^{2}\right)-\mu c s$.

Relations (31) define a domain of the $(\alpha, \beta)$ plane, therefore of the $\left(R_{1 n}, R_{2 n}\right)$ plane. It is easy to see that if $\mu<\left(1+c^{2}\right) / c s$ and either $d_{1}$ or $d_{2}$ are negative, then the domain defined by (31) is empty. We have represented in Figs. 8 and 9 the different situations that can occur when both $d_{1}$ and $d_{2}$ are strictly positive according to different values of $\mu$. The values of $\left(R_{1 n}, R_{2 n}\right)$ on the boundary of the domain correspond to equilibrium solutions that are in imminent sliding. We observe that the shape of the $\left(R_{1 n}, R_{2 n}\right)$ equilibria domain depends on the parameters and the domains may be unbounded.

To summarize the results given in this section we can say that for any given sign of the data $A_{1}, A_{2}, d_{1}, d_{2}$ and according to whether $\mu$ is smaller 
Fig. 8 Bounded domains of equilibrium in the $\alpha\left(=R_{1 n}\right), \beta\left(=R_{2 n}\right)$ plane
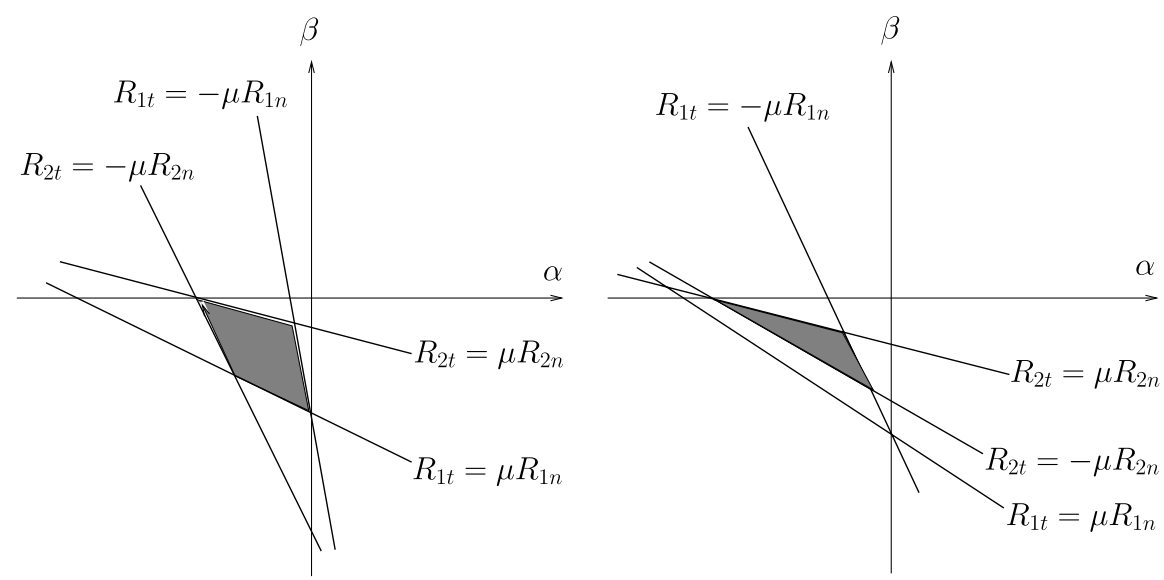

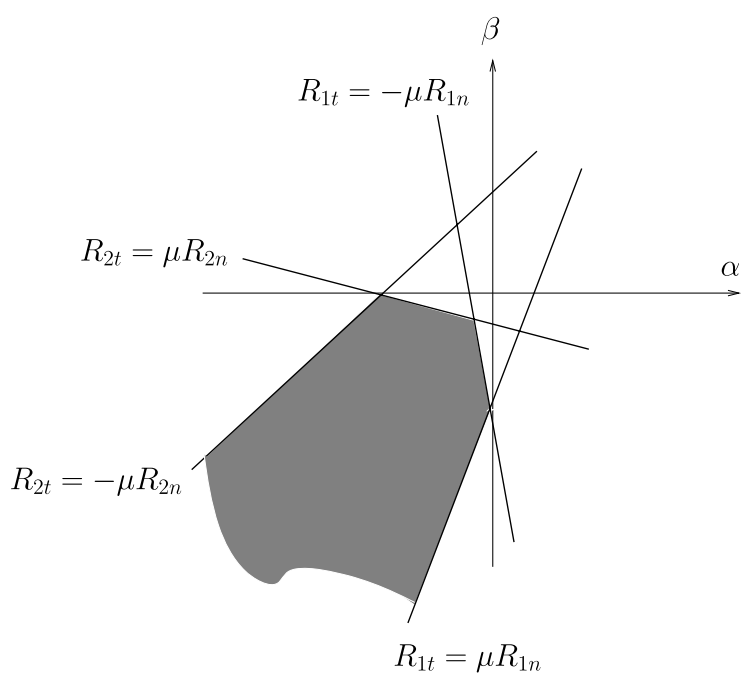

Fig. 9 Unbounded domains of equilibrium in the $\alpha\left(=R_{1 n}\right)$, $\beta\left(=R_{2 n}\right)$ plane

than $3 c / 2 s$, greater than $\left(1+c^{2}\right) / c s$ or between both values, we can explicitly determine the possible equilibrium solutions. In general, equilibrium solutions where only one mass is in contact and solutions where both masses are in contact, coexist. However, when $A_{1}<0, A_{2}<0$ and $\mu<3 c / 2 s$, the only possible equilibrium solution is the solution where the two masses are not in contact. When $A_{1}=0$ and $A_{2}=0$ and $\mu<3 c / 2 s$, the only equilibrium solution is the one where the two masses are in grazing contact.

It is interesting to note the complexity of the results obtained here compared to those obtained in the case of a single mass. We could in the case of a single mass represent the nine different sets of equilibrium solutions according to the values of the data (see [5]). Here there would be more than thirty different sets of equilibrium solutions and inside a given set one could have an infinity of solutions where the two masses are in contact, together with another infinity where only one mass is in contact. There is no point in giving all these different sets, we need simply to be able to determine the set corresponding to a given data through the analysis given in this section.

\subsection{Stability}

Having obtained a complete description of the equilibrium solutions, we proceed in our endeavor to back up the conjecture given in the Introduction.

In the two-mass case the conjecture concerns a perturbation of an equilibrium where the two masses are strictly in contact. If one of the two masses is not in contact, the motion of the two masses obtained by perturbing the mass which is strictly in contact can be explicitly computed and one can check that after a sliding of the mass in contact (or eventually several right and left slidings) the mass in contact shall stay motionless, whereas the mass which is not in contact shall oscillate indefinitely. So that a new equilibrium state is never reached. If both reactions are strictly inside the Coulomb cone then there exists small enough perturbation such that the reactions stay strictly inside the Coulomb cone, in which case the conjecture is trivial. Consequently, the result remains to be established only in the case where at least one mass is in imminent sliding. We start by studying the case where only one mass is set in motion by the perturbation before studying the case where both masses are set in motion. 


\subsubsection{One mass is stuck, the other is in imminent sliding}

We come back to problem (11)-(15) with initial data corresponding to an equilibrium where only one mass is in imminent sliding. A constant perturbation $\varepsilon$ of the force is applied, and we want to show that the trajectory leads to a new equilibrium where both reactions are strictly inside the cone. We compute the normal reactions of mass 1 and mass 2 corresponding to an equilibrium solution with the reaction of mass 1 strictly inside the Coulomb cone and mass 2 in imminent sliding towards the right (for example, when the parameters correspond to the reactions represented in Fig. 8). Such an equilibrium solution satisfies $u_{1 n}=u_{2 n}=0, u_{1 t}=\bar{u}_{1 t}, u_{2 t}=\bar{u}_{2 t}, R_{1 n}=R_{1 n}^{\mathrm{eq}}$, $R_{2 n}=R_{2 n}^{\mathrm{eq}},\left|R_{1 t}^{\mathrm{eq}}\right|<-\mu R_{1 n}^{\mathrm{eq}}$ and $R_{2 t}^{\mathrm{eq}}=\mu R_{2 n}^{\mathrm{eq}}$, where:

$\bar{u}_{1 t}=\frac{F_{1 n}+R_{1 n}^{\mathrm{eq}}}{s c}, \quad \bar{u}_{2 t}=-\frac{F_{2 n}+R_{2 n}^{\mathrm{eq}}}{s c}=\frac{\mathcal{D}}{\mathcal{C}}$

with $\mathcal{C}$ defined above in (32) and $\mathcal{D}$ defined by:

$\mathcal{D}=F_{2 t}-\mu F_{2 n}+\frac{F_{1 n}+R_{1 n}^{\mathrm{eq}}}{s c}$.

For such an equilibrium solution, both $R_{1 n}^{\mathrm{eq}}$ and $R_{2 n}^{\mathrm{eq}}$ are strictly negative and we suppose that the perturbation $\varepsilon$ is sufficiently small to ensure that the normal reactions of both masses remain strictly negative and that the reaction of mass 1 stays strictly inside the Coulomb cone. As the equilibrium solution we consider in this subsection is such that the reaction of mass 1 is strictly inside the Coulomb cone and mass 2 is in imminent sliding, by (31) and (32), $R_{1 n}^{\mathrm{eq}}$ and $R_{2 n}^{\mathrm{eq}}$ must satisfy the following relations:

$$
\left\{\begin{array}{l}
d_{1}+\mathcal{C} R_{1 n}^{\mathrm{eq}}+R_{2 n}^{\mathrm{eq}}<0 \\
d_{1}+\mathcal{C}^{\prime} R_{1 n}^{\mathrm{eq}}+R_{2 n}^{\mathrm{eq}}>0 \\
d_{2}+\mathcal{C} R_{2 n}^{\mathrm{eq}}+R_{1 n}^{\mathrm{eq}}=0 \\
d_{2}+\mathcal{C}^{\prime} R_{2 n}^{\mathrm{eq}}+R_{1 n}^{\mathrm{eq}}>0
\end{array}\right.
$$

If we perturb $F_{2 n}$ by some positive constant $\varepsilon$ then the reaction of mass 2 is strictly inside the Coulomb cone and mass 2 stays motionless. But if we add $\varepsilon<0$ to
$F_{2 n}$, then problem (11)-(15) becomes:

$$
\left\{\begin{array}{l}
u_{1 t}(t)=\bar{u}_{1 t}, u_{1 n}(t)=0, u_{2 n}(t)=0 \quad \forall t \\
\ddot{u}_{2 t}+\mathcal{C} u_{2 t}=F_{2 t}-\mu\left(F_{2 n}+\varepsilon\right)+\frac{F_{1 n}+R_{1 n}^{\mathrm{eq}}}{s c} \\
=\mathcal{D}-\mu \varepsilon, \\
u_{2 t}(0)=\bar{u}_{2 t}=\frac{\mathcal{D}}{\mathcal{C}}, \\
\dot{u}_{2 t}(0)=0 ;
\end{array}\right.
$$

therefore

$u_{2 t}(t)=\frac{\mathcal{D}-\mu \varepsilon}{\mathcal{C}}+\frac{\mu \varepsilon}{\mathcal{C}} \cos \sqrt{\mathcal{C}} t$.

We are going to show that when mass 2 stops sliding the system reaches a new equilibrium where the reactions of both masses are strictly inside the Coulomb cone.

Let $\tilde{t}>0$ be such that $\dot{u}_{2 t}(\tilde{t})=0$ (i.e. $\tilde{t}$ is the instant when mass 2 stops sliding); then $u_{2 t}(\tilde{t})=\frac{\mathcal{D}-2 \mu \varepsilon}{\mathcal{C}}$. As mass 2 is in bilateral contact, here Coulomb's law and the equations of motion imply that there is no velocity jump at $t=\tilde{t}$ (see [4]). So that $\dot{u}_{2 t}\left(\tilde{t}^{+}\right)=0$. We shall now show that there exists $\eta$ such that $\dot{u}_{2 t}(t)=0 \forall t \in$ ]$\tilde{t}, \tilde{t}+\eta[$; in other words, that mass 2 stays motionless after $\tilde{t}$.

Let us suppose that we have $\left.\dot{u}_{2 t}(t)>0 \forall t \in\right] \tilde{t}, \tilde{t}+$ $\eta[$. Then mass 2 continues to slide to the right (i.e. $\left.R_{2 t}=\mu R_{2 n}\right)$. Consequently, the motion is still described by system (34) in ] $\tilde{t}, \tilde{t}+\eta[$ and there is no jump of the tangential component of the acceleration of mass 2 . We therefore have $\ddot{u}_{2 t}(\tilde{t})=\mu \varepsilon<0$ and thus $\dot{u}_{2 t}(t)<0$ for $\left.t \in\right] \tilde{t}, \tilde{t}+\eta[$, which contradicts the assumption.

Let us now assume that $\dot{u}_{2 t}(t)<0$ for $\left.t \in\right] \tilde{t}, \tilde{t}+\eta[$. Then mass 2 will slide to the left (i.e. $R_{2 t}=-\mu R_{2 n}$ ) and $u_{2 t}$ satisfies the following system for $\left.t \in\right] \tilde{t}, \tilde{t}+\eta[$ :

$\left\{\begin{array}{l}\ddot{u}_{2 t}+\mathcal{C}^{\prime} u_{2 t}=\mathcal{D}^{\prime}+\mu \varepsilon, \\ u_{2 t}(\tilde{t})=\frac{\mathcal{D}-2 \mu \varepsilon}{\mathcal{C}}, \quad \dot{u}_{2 t}(\tilde{t})=0,\end{array}\right.$

where $\mathcal{D}^{\prime}=F_{2 t}+\mu F_{2 n}+\bar{u}_{1 t}$.

So that we have $\ddot{u}_{2 t}\left(\tilde{t}^{+}\right)=-\frac{1}{\mathcal{C}}\left(\mathcal{C}^{\prime}(\mathcal{D}-2 \mu \varepsilon)-\right.$ $\left.\mathcal{C}\left(\mathcal{D}^{\prime}+\mu \varepsilon\right)\right)$.

But as $\mathcal{C}^{\prime}(\mathcal{D}-2 \mu \varepsilon)-\mathcal{C}\left(\mathcal{D}^{\prime}+\mu \varepsilon\right)=2 \mu \mathcal{C} R_{2 n}^{\mathrm{eq}}-$ $\left(3\left(1+c^{2}\right)-\mu c s\right) \mu \varepsilon$, there exists $\varepsilon_{0}>0$ such that if $|\varepsilon|<\varepsilon_{0}$ then this quantity is strictly negative and 
$\ddot{u}_{2 t}\left(\tilde{t}^{+}\right)>0$. Therefore $\dot{u}_{2 t}$ is positive in some interval ]$\tilde{t}, \tilde{t}+\eta[$, which contradicts the assumption.

We can now conclude that for $t \in] \tilde{t}, \tilde{t}+\eta[$ we have $\dot{u}_{2 t}(t)=0$, so that the displacements and reactions are solution to system (16) in this time interval, which implies that at time $\tilde{t}$ there is a jump in the tangential reaction of mass 2 and in its tangential acceleration.

We have: $\ddot{u}_{2 t}\left(\tilde{t}^{+}\right)=0$ and $\tilde{R}_{2 t}^{+}=R_{2 t}^{\mathrm{eq}}-2 \mu \frac{1+c^{2}}{\mathcal{C}} \varepsilon$.

If we now compute the normal reaction of mass 2 at $\tilde{t}$,

$$
\begin{aligned}
\tilde{R}_{2 n} & =R_{2 n}(\tilde{t})=-\operatorname{csu}_{2 t}(\tilde{t})-F_{2 n}-\varepsilon \\
& =R_{2 n}^{\mathrm{eq}}-\varepsilon \frac{\mathcal{C}^{\prime}}{\mathcal{C}},
\end{aligned}
$$

and a simple computation ensures that there exists $\varepsilon_{0}>0$ such that if $|\varepsilon|<\varepsilon_{0}$, then $R_{1 n}^{\mathrm{eq}}$ and $\tilde{R}_{2 n}$ satisfy the following relations:

$$
\left\{\begin{array}{l}
\tilde{d}_{1}+\mathcal{C} R_{1 n}^{\mathrm{eq}}+\tilde{R}_{2 n}<0, \\
\tilde{d}_{1}+\mathcal{C}^{\prime} R_{1 n}^{\mathrm{eq}}+\tilde{R}_{2 n}>0, \\
\tilde{d}_{2}+\mathcal{C} \tilde{R}_{2 n}+R_{1 n}^{\mathrm{eq}}<0, \\
\tilde{d}_{2}+\mathcal{C}^{\prime} \tilde{R}_{2 n}+R_{1 n}^{\mathrm{eq}}>0,
\end{array}\right.
$$

where $\tilde{d}_{1}=d_{1}+\varepsilon$ and $\tilde{d}_{2}=d_{2}+\left(1+c^{2}\right) \varepsilon$.

This proves the conjecture when mass 2 is in imminent sliding to the right. When mass 2 is in imminent sliding to the left, if we perturb $F_{2 n}$ by adding $\varepsilon>0$ then the reactions of mass 2 are strictly inside the Coulomb cone and mass 2 stays motionless, but if we add $\varepsilon<0$ to $F_{2 n}$ then mass 2 starts to slide to the left. A similar computation to the one given above establishes that there exists $\varepsilon_{0}>0$ such that if $|\varepsilon|<\varepsilon_{0}$ then when mass 2 stops sliding to the left its reaction is strictly inside the Coulomb cone and therefore that mass 2 stays motionless. Note that we have here applied the perturbation to the normal component of the loading but it is easy to check that we have the same result when applying the perturbation to the tangential component. We shall therefore continue, in this section, to apply the perturbation to the normal components of the loading.

\subsubsection{Perturbing both masses in imminent sliding}

When both masses are in imminent sliding, one to the left, the other to the right, applying a positive perturbation to the normal loading of mass 2 leaves the reaction of mass 2 strictly inside the Coulomb cone and mass 1 in imminent sliding. Whereas a negative perturbation initiates the sliding of mass 2 and this sliding makes the reaction of mass 1 lie strictly inside the Coulomb cone.

In this subsection we are going to add a perturbation to the normal loading of both masses who are both in imminent sliding. We suppose that mass 1 is in imminent left sliding and mass 2 in imminent right sliding so that we have:

$d_{1}+\mathcal{C} R_{1 n}^{\mathrm{eq}}+R_{2 n}^{\mathrm{eq}}=0 \quad$ and $\quad d_{2}+\mathcal{C} R_{2 n}^{\mathrm{eq}}+R_{1 n}^{\mathrm{eq}}=0$.

Adding $\varepsilon_{1}$ to the loading $F_{1 n}$ and $\varepsilon_{2}$ to the loading $F_{2 n}$ changes the parameters $d_{1}$ and $d_{2}$ that take the following values:

$\tilde{d}_{1}=d_{1}+\left(1+c^{2}\right) \varepsilon_{1}+\varepsilon_{2} \quad$ and

$\tilde{d}_{2}=d_{2}+\varepsilon_{1}+\left(1+c^{2}\right) \varepsilon_{2}$.

So that the normal reactions become after the perturbation:

$R_{1 n}(0)=R_{1 n}^{\mathrm{eq}}-\varepsilon_{1} \quad$ and $\quad R_{2 n}(0)=R_{2 n}^{\mathrm{eq}}-\varepsilon_{2}$.

Therefore

$\tilde{d}_{1}+\mathcal{C} R_{1 n}(0)+R_{2 n}(0)=-\mu \operatorname{cs} \varepsilon_{1}$,

$\tilde{d}_{2}+\mathcal{C} R_{2 n}(0)+R_{1 n}(0)=-\mu \operatorname{cs} \varepsilon_{2}$.

From these expressions we see that if $\varepsilon_{1}$ is positive, the reaction of mass 1 is in the Coulomb cone and if $\varepsilon_{2}$ is positive, the reaction of mass 2 is in the Coulomb cone. Moreover, as the case when only one perturbation is negative has been studied in the preceding subsection, we suppose here that both $\varepsilon_{1}$ and $\varepsilon_{2}$ are negative.

The motion of the two masses satisfies the following system:

$\left\{\begin{array}{l}\ddot{u}_{1 t}+\mathcal{C} u_{1 t}-u_{2 t}=F_{1 t}+\mu F_{1 n}+\mu \varepsilon_{1}, \\ \ddot{u}_{2 t}-u_{1 t}+\mathcal{C} u_{2 t}=F_{2 t}-\mu F_{2 n}-\mu \varepsilon_{2}, \\ u_{1 t}(0)=\bar{u}_{1 t}=\frac{\mathcal{C}\left(F_{1 t}+\mu F_{1 n}\right)+F_{2 t}-\mu F_{2 n}}{\mathcal{C}^{2}-1}, \\ \dot{u}_{1 t}(0)=0, \\ u_{2 t}(0)=\bar{u}_{2 t}=\frac{\mathcal{C}\left(F_{2 t}-\mu F_{2 n}\right)+F_{1 t}+\mu F_{1 n}}{\mathcal{C}^{2}-1}, \\ \dot{u}_{2 t}(0)=0 .\end{array}\right.$ 
And therefore we have:

$$
\begin{aligned}
u_{1 t}(t)= & \bar{u}_{1 t}+\mu \frac{\mathcal{C} \varepsilon_{1}-\varepsilon_{2}}{\mathcal{C}^{2}-1}+\mu \frac{\varepsilon_{2}-\varepsilon_{1}}{2 \omega_{1}^{2}} \cos \omega_{1} t \\
& -\mu \frac{\varepsilon_{1}+\varepsilon_{2}}{2 \omega_{2}^{2}} \cos \omega_{2} t, \\
u_{2 t}(t)= & \bar{u}_{2 t}+\mu \frac{\varepsilon_{1}-\mathcal{C} \varepsilon_{2}}{\mathcal{C}^{2}-1}+\mu \frac{\varepsilon_{2}-\varepsilon_{1}}{2 \omega_{1}^{2}} \cos \omega_{1} t \\
& +\mu \frac{\varepsilon_{1}+\varepsilon_{2}}{2 \omega_{2}^{2}} \cos \omega_{2} t . \\
\dot{u}_{1 t}(t)= & -\frac{\mu}{2 \omega_{1}}\left(\varepsilon_{2}-\varepsilon_{1}\right) \sin \omega_{1} t \\
& +\frac{\mu}{2 \omega_{2}}\left(\varepsilon_{1}+\varepsilon_{2}\right) \sin \omega_{2} t, \\
\dot{u}_{2 t}(t)= & -\frac{\mu}{2 \omega_{1}}\left(\varepsilon_{2}-\varepsilon_{1}\right) \sin \omega_{1} t \\
& -\frac{\mu}{2 \omega_{2}}\left(\varepsilon_{1}+\varepsilon_{2}\right) \sin \omega_{2} t,
\end{aligned}
$$

where $\omega_{1}=\sqrt{\mathcal{C}-1}$ and $\omega_{2}=\sqrt{\mathcal{C}+1}$.

If $\varepsilon_{1}$ is different from $\varepsilon_{2}$, let $\varepsilon_{1}<\varepsilon_{2}<0$. In this case mass 2 stops before mass 1 . Let $\tilde{t}$ be such that $\dot{u}_{2 t}(\tilde{t})=0$ and $\dot{u}_{2 t}(t)>0$ for $0<t<\tilde{t}$. We are going to prove that the further dynamics is such that $\dot{u}_{2 t}(t)=0 \forall t>\tilde{t}$. Assume there exists $\eta$ such that $\left.\dot{u}_{2 t}(t)>0 \forall t \in\right] \tilde{t}, \tilde{t}+\eta[$, then mass 2 continues to slide to the right and the motion continues to satisfy system (39) so that, as the corresponding solution is as smooth as we want in $] \tilde{t}-\eta, \tilde{t}+\eta[$,

$\dddot{u}_{2 t}(\tilde{t})=\frac{\mu}{2}\left(\omega_{1}\left(\varepsilon_{2}-\varepsilon_{1}\right) \sin \omega_{1} \tilde{t}+\omega_{2}\left(\varepsilon_{1}+\varepsilon_{2}\right) \sin \omega_{2} \tilde{t}\right)$,

but $\dot{u}_{2 t}(\tilde{t})=0 \Longrightarrow \frac{\varepsilon_{1}+\varepsilon_{2}}{\omega_{2}} \sin \omega_{2} \tilde{t}=-\frac{\varepsilon_{2}-\varepsilon_{1}}{\omega_{1}} \sin \omega_{1} \tilde{t}$ then

$\dddot{u}_{2 t}(\tilde{t})=-\mu \frac{\varepsilon_{2}-\varepsilon_{1}}{\omega_{1}} \sin \omega_{1} \tilde{t}$.

Inserting $\bar{t}=\frac{\pi}{\omega_{2}}$ into (41) we obtain that $\dot{u}_{2 t}(\bar{t})<0$ because $\omega_{1}<\omega_{2}$; this implies that $\tilde{t}<\bar{t}=\frac{\pi}{\omega_{2}}$ so that $\dddot{u}_{2 t}(\tilde{t})<0$ and $\left.\dot{u}_{2 t}(t)<0 \forall t \in\right] \tilde{t}, \tilde{t}+\eta[$. This contradicts the assumption that $\left.\dot{u}_{2 t}(t)>0 \forall t \in\right] \tilde{t}, \tilde{t}+\eta[$.

Let us now assume $\left.\dot{u}_{2 t}(t)>0 \forall t \in\right] \tilde{t}, \tilde{t}+\eta[$, then mass 2 slides to the left and the motion of the two masses satisfies the following system:

$$
\left\{\begin{array}{l}
\ddot{u}_{1 t}+\mathcal{C} u_{1 t}-u_{2 t}=F_{1 t}+\mu F_{1 n}+\mu \varepsilon_{1} \stackrel{\text { def }}{\equiv} \tilde{F}_{1}, \\
\ddot{u}_{2 t}-u_{1 t}+\mathcal{C}^{\prime} u_{2 t}=F_{2 t}+\mu F_{2 n}+\mu \varepsilon_{2} \stackrel{\text { def }}{\equiv} \tilde{F}_{2} .
\end{array}\right.
$$

For example, when $\mathcal{C C}^{\prime}-1>0$, the solution of this system is given by:

$$
\left\{\begin{aligned}
u_{1 t}(t)= & \frac{\mathcal{C}^{\prime} \tilde{F}_{1}+\tilde{F}_{2}}{\mathcal{C} \mathcal{C}^{\prime}-1}+c_{1}\left(\mathcal{C}^{\prime}-\gamma_{1}^{2}\right) \cos \gamma_{1}(t-\tilde{t}) \\
& +c_{2}\left(\mathcal{C}^{\prime}-\gamma_{1}^{2}\right) \sin \gamma_{1}(t-\tilde{t}) \\
& +c_{3}\left(\mathcal{C}^{\prime}-\gamma_{2}^{2}\right) \cos \gamma_{2}(t-\tilde{t}) \\
& +c_{4}\left(\mathcal{C}^{\prime}-\gamma_{2}^{2}\right) \sin \gamma_{2}(t-\tilde{t}) \\
u_{2 t}(t)= & \frac{\mathcal{C} \tilde{F}_{2}+\tilde{F}_{1}}{\mathcal{C} \mathcal{C}^{\prime}-1}+c_{1} \cos \gamma_{1}(t-\tilde{t})+c_{2} \sin \gamma_{1}(t-\tilde{t}) \\
& +c_{3} \cos \gamma_{2}(t-\tilde{t})+c_{4} \sin \gamma_{2}(t-\tilde{t}),
\end{aligned}\right.
$$

where

$$
\begin{aligned}
& \gamma_{1}=\sqrt{\frac{\left(\mathcal{C}+\mathcal{C}^{\prime}\right)-\sqrt{\left(\mathcal{C}-\mathcal{C}^{\prime}\right)^{2}+4}}{2}} \text { and } \\
& \gamma_{2}=\sqrt{\frac{\left(\mathcal{C}+\mathcal{C}^{\prime}\right)+\sqrt{\left(\mathcal{C}-\mathcal{C}^{\prime}\right)^{2}+4}}{2}} .
\end{aligned}
$$

Then, from (43), we have $\ddot{u}_{2 t}\left(\tilde{t}^{+}\right)=-c_{1} \gamma_{1}^{2}-c_{3} \gamma_{2}^{2}$. But whatever the sign of $\mathcal{C C}^{\prime}-1$, we always have:

$$
\begin{aligned}
\ddot{u}_{2 t}\left(\tilde{t}^{+}\right)= & u_{1 t}(\tilde{t})-\mathcal{C}^{\prime} u_{2 t}(\tilde{t}) \\
& -\frac{\mathcal{C}^{\prime} \tilde{F}_{1}+\tilde{F}_{2}}{\mathcal{C} \mathcal{C}^{\prime}-1}+\mathcal{C}^{\prime} \frac{\mathcal{C} \tilde{F}_{2}+\tilde{F}_{1}}{\mathcal{C} \mathcal{C}^{\prime}-1} \\
= & u_{1 t}(\tilde{t})-\mathcal{C}^{\prime} u_{2 t}(\tilde{t})+\tilde{F}_{2} .
\end{aligned}
$$

And finally we have:

$$
\begin{aligned}
\ddot{u}_{2 t}\left(\tilde{t}^{+}\right)= & F_{2 t}+\mu F_{2 n}+\mu \varepsilon_{2}+\bar{u}_{1 t}-\mathcal{C}^{\prime} \bar{u}_{2 t} \\
& +\frac{\mu\left(\mathcal{C} \varepsilon_{1}-\varepsilon_{2}-\mathcal{C}^{\prime} \varepsilon_{1}+\mathcal{C C}^{\prime} \varepsilon_{2}\right)}{\mathcal{C}^{2}-1} \\
& +\frac{\mu}{2 \omega_{1}^{2}}\left(1-\mathcal{C}^{\prime}\right)\left(\varepsilon_{2}-\varepsilon_{1}\right) \cos \omega_{1} \tilde{t} \\
& -\frac{\mu}{2 \omega_{2}^{2}}\left(1+\mathcal{C}^{\prime}\right)\left(\varepsilon_{2}+\varepsilon_{1}\right) \cos \omega_{2} \tilde{t}
\end{aligned}
$$


with

$\bar{u}_{1 t}=\frac{1}{c s}\left(F_{1 n}+R_{1 n}^{\mathrm{eq}}\right), \quad \bar{u}_{2 t}=-\frac{1}{c s}\left(F_{2 n}+R_{2 n}^{\mathrm{eq}}\right)$.

We observe that $\ddot{u}_{2 t}\left(\tilde{t}^{+}\right)$is of the form

$\ddot{u}_{2 t}\left(\tilde{t}^{+}\right)=\alpha+\beta \varepsilon_{1}+\delta \varepsilon_{2}$.

As a function of $\varepsilon_{1}$ and $\varepsilon_{2}$, it is immediately seen that

$\alpha>0 \quad \Longrightarrow \quad \exists \varepsilon_{0}>0$

such that

$\left|\varepsilon_{1}\right|<\varepsilon_{0} \quad$ and $\quad\left|\varepsilon_{2}\right|<\varepsilon_{0} \quad \Longrightarrow \quad \ddot{u}_{2 t}\left(\tilde{t}^{+}\right)>0$.

We thus have:

$$
\begin{aligned}
\alpha= & F_{2 t}+\mu F_{2 n} \\
& +\frac{1}{c s}\left(F_{1 n}+R_{1 n}^{\mathrm{eq}}\right)+\mathcal{C}^{\prime} \frac{1}{c s}\left(F_{2 n}+R_{2 n}^{\mathrm{eq}}\right)
\end{aligned}
$$

and finally:

$$
\begin{aligned}
\alpha & =\frac{1}{c s}\left(d_{2}+R_{1 n}^{\mathrm{eq}}+\mathcal{C} R_{2 n}^{\mathrm{eq}}-2 \mu c s R_{2 n}^{\mathrm{eq}}\right) \\
& =-2 \mu R_{2 n}^{\mathrm{eq}}>0 .
\end{aligned}
$$

We have therefore shown that mass 2 stays motionless for some time after $\tilde{t}$. However, mass 1 continues to move and to affect the reaction of mass 2. Does the reaction of mass 2 stay strictly inside the Coulomb cone for all time after $\tilde{t}$ ? To check that, we must compute the tangential component of the reaction of mass 2 . At $\tilde{t}^{+}$the reaction of mass 2 is given by:

$\tilde{R}_{2 n}=-\operatorname{cs} \tilde{u}_{2 t}-F_{2 n}-\varepsilon_{2}$,

$\tilde{R}_{2 t}=-\tilde{u}_{1 t}+\left(1+c^{2}\right) \tilde{u}_{2 t}-F_{2 t}$,

and because its reaction at time $\tilde{t}$ is strictly inside the Coulomb cone, we know that the following inequality holds:

$\mu \tilde{R}_{2 n}<\tilde{R}_{2 t}<-\mu \tilde{R}_{2 n}$.

For $t>\tilde{t}$ the motion of mass 1 is given by:

$$
\begin{aligned}
u_{1 t}(t)= & \tilde{u}_{1 t}+\left(\frac{U}{\mathcal{C}}-\tilde{u}_{1 t}\right)(1-\cos \sqrt{\mathcal{C}}(t-\tilde{t})) \\
& +\frac{\tilde{\dot{u}}_{1 t}}{\sqrt{\mathcal{C}}} \sin \sqrt{\mathcal{C}}(t-\tilde{t})
\end{aligned}
$$

where

$U=F_{1 t}+\mu F_{1 n}+\mu \varepsilon_{1}+\tilde{u}_{2 t}$,

$\tilde{u}_{1 t}=u_{1 t}(\tilde{t}) \quad$ and $\quad \tilde{\dot{u}}_{1 t}=\dot{u}_{1 t}(\tilde{t})<0$.

We therefore have:

$$
\begin{aligned}
R_{2 t}= & \tilde{R}_{2 t}-\left(\frac{U}{\mathcal{C}}-\tilde{u}_{1 t}\right)(1-\cos \sqrt{\mathcal{C}}(t-\tilde{t})) \\
& -\frac{\tilde{\dot{u}}_{1 t}}{\sqrt{\mathcal{C}}} \sin \sqrt{\mathcal{C}}(t-\tilde{t})
\end{aligned}
$$

but

$\tilde{\dot{u}}_{1 t}=\frac{\mu}{\omega_{2}}\left(\varepsilon_{1}+\varepsilon_{2}\right) \sin \omega_{2} \tilde{t}$,

and

$\frac{U}{\mathcal{C}}-\tilde{u}_{1 t}=\frac{\mu}{2}\left(\varepsilon_{1}-\varepsilon_{2}\right) \cos \omega_{1} \tilde{t}+\frac{\mu}{2}\left(\varepsilon_{1}+\varepsilon_{2}\right) \cos \omega_{2} \tilde{t}$.

So that

$$
\begin{aligned}
\left|R_{2 t}-\tilde{R}_{2 t}\right| \leq & \left|\frac{\mu}{\omega_{2} \sqrt{\mathcal{C}}}\left(\varepsilon_{1}+\varepsilon_{2}\right)\right| \\
& +\left|\mu\left(\varepsilon_{1}-\varepsilon_{2}\right)\right|+\left|\mu\left(\varepsilon_{1}+\varepsilon_{2}\right)\right| .
\end{aligned}
$$

We can conclude that if $\varepsilon_{1}$ and $\varepsilon_{2}$ are sufficiently small, the reaction of mass 2 always stays strictly inside the Coulomb cone. So that the motion of mass 1 can never put mass 2 back into motion. When in turn mass 1 stops, the two masses are in a strictly stuck equilibrium. Once again, this result backs up our conjecture.

\section{Numerical experiments on a finite element discretization of an elastic body}

This section contains a numerical computation of the trajectory of a system with a much larger number of degrees of freedom than the systems studied analytically in the preceding sections. The model is obtained by finite element discretization of a rectangular elastic bloc. We insist here on the fact that we do not intend to compute the trajectory of a continuous media with unilateral contact and Coulomb friction. Indeed, in such a case all the problems relating to continuum mechanics, in particular the convergence of the discretization, are open and difficult ones. Therefore the following 
Fig. 10 The $30 \times 10 Q 4$ meshed block

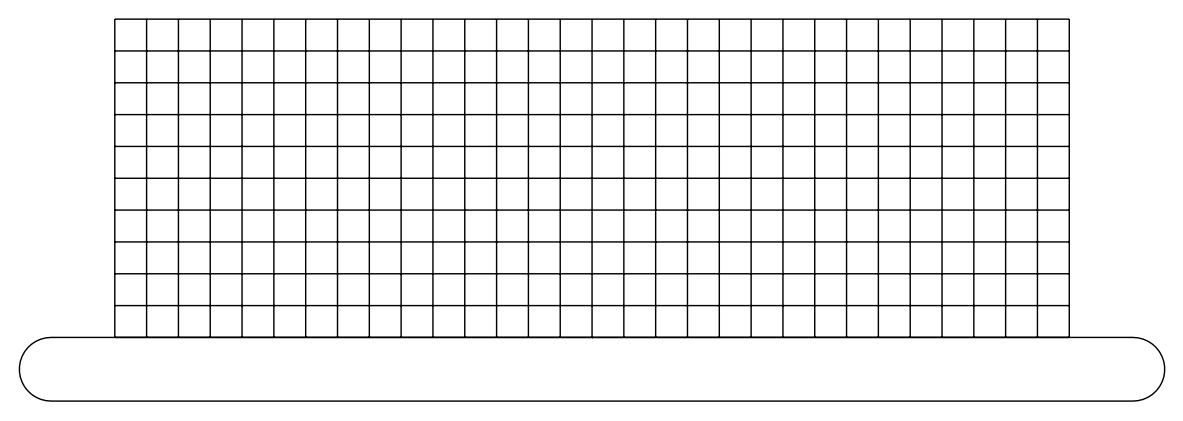

Fig. 11 The quasistatically deformed block at equilibrium and the reaction forces at the nodes

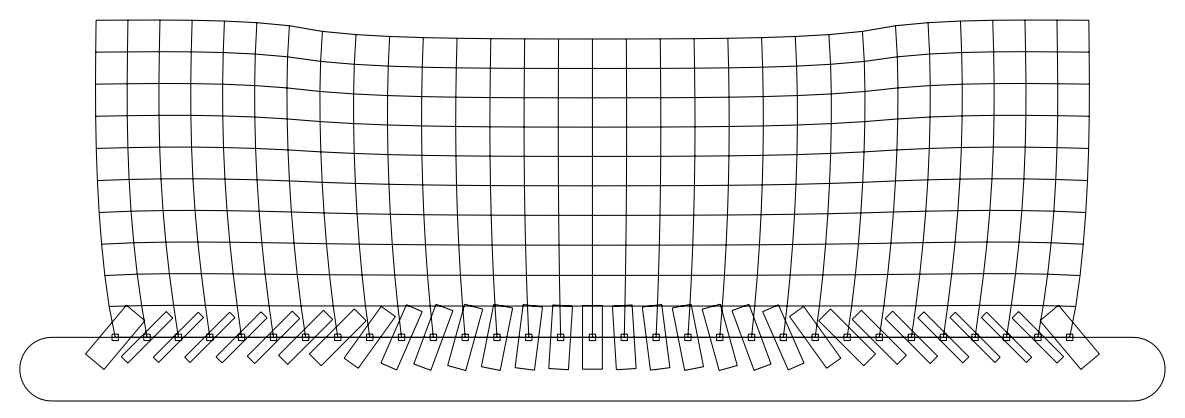

computation must be viewed as a model generalizing, through the number of degrees of freedom, the one- or two-mass systems studied in the preceding sections. In fact, Klarbring's model has often been said to represent the behavior of a finite element mesh.

The computations are made using the Non-Smooth Contact Dynamics method [8], implemented in the software LMGC90 (see [7]). A 2-dimensional elastic block is meshed with $30 \times 10 Q 4$ square elements, of length $l=10^{-3} \mathrm{~m}$. The material is linearly elastic in small perturbations with the following properties: mass per unit volume $\rho=10^{4} \mathrm{~kg} / \mathrm{m}^{3}$, Young modulus $E=5 \times 10^{6} \mathrm{~Pa}$, Poisson coefficient $v=0.49$, so that the celerity is $C=\sqrt{E / \rho}=0.707 \times 10^{2} \mathrm{~m} / \mathrm{s}$. The propagation time through a mesh element is $\tau=$ $l / C=0.14 \times 10^{-4} \mathrm{~s}$. Plane deformations are assumed. The block is lying on the plane face of some fixed rigid object, referred to as the foundation, see Fig. 10. The candidates to contact are numbered from 1 to 31 , from left to right. Coulomb's friction is assumed between the block and the foundation with a friction coefficient $\mu=1.0$. The collection of nodes of the upper layer is numbered from $1 \times 11$ to $31 \times 11$, from left to right. Each node numbered $8 \times 11$ to $24 \times 11$ is submitted to a vertical force $-0.75 \times 10^{3} \mathrm{~N}$. The gravity forces are neglected.

\subsection{A reference state with imminent sliding contacts}

A dynamical computation is performed, using a time step of $0.2 \times 10^{-2} \mathrm{~s}$, which is quite larger than $\tau=$ $0.14 \times 10^{-4} \mathrm{~s}$, so that a quasistatic evolution is computed practically at once. The computation yields the following equilibrium response displayed in Fig. 11, symmetric with respect to the vertical axis of the block (node 15, node 166). In this figure, reaction forces at contacting points are represented by rectangles, the longer side being directed as the reaction force, and the width of the rectangle being proportional to the force modulus $\left(R_{t}^{2}+R_{n}^{2}\right)^{\frac{1}{2}}$. The nodes $2,3,4,5,6,7,8$, and 24, 25, 26, 27, 28, 29, 30 are imminent sliding contacts (within an accuracy range of $10^{-3}$ ).

The $\left|R_{t}\right| / \mu R_{n}$ distribution is displayed in Fig. 12 . This distribution is constructed as follows. Let $\chi \in$ $[0,1]$ be a real number and let $N$ be the total number of contacts ${ }^{\alpha}$ where $\mu R_{n}^{\alpha}$ is different from zero (here $N=31$ ); let $N(\chi)$ be the number of contacts where $\left|R_{t}^{\alpha}\right| / \mu R_{n}^{\alpha} \geq \chi$; the $\left|R_{t}\right| / \mu R_{n}$ distribution is defined as the function $\chi \rightarrow N(\chi) / N$. This function is decreasing from 1 to some positive value, which is 0 if the sample is at equilibrium in a Coulomb stable situation. In this example, there are 14 imminent sliding contacts, i.e. a ratio of 0.45 . 


\subsection{The block perturbed by some horizontal shock}

The block is considered at the equilibrium state obtained by the quasistatic evolution above. A perturbation is applied generated by the impact of a light rigid projectile as depicted in Fig. 13. This rigid projectile is

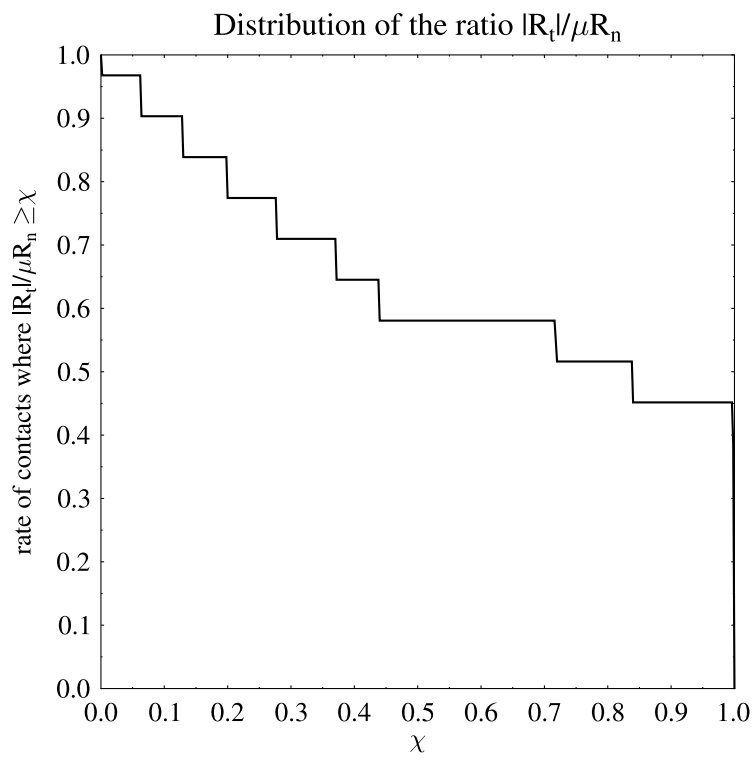

Fig. 12 The $\left|R_{t}\right| / \mu R_{n}$ distribution of the quasistatically deformed block thrown on the left side of the block with an horizontal velocity equal to $3 \mathrm{~m} / \mathrm{s}$. The mass of the projectile is about $2.6 \%$ of the mass of the block.

Here the time step is chosen so as to capture correctly dynamical evolutions. The time step is of $0.1 \times$ $10^{-5} \mathrm{~s}$, which is smaller therefore than the time of propagation $\tau$ within a mesh element $\tau=l / C=$ $0.14 \times 10^{-4} \mathrm{~s}$. After the episode of impact the projectile is thrown backward and a complex system of waves is generated (see Fig. 16) displaying the nodes velocity field at $0.2 \times 10^{-3} \mathrm{~s}$, after the projectile has bounced off the block. It may be observed that the amplitude of waves is decaying near the bottom of the block in contact with the foundation. A pure longitudinal wave would take approximately $0.84 \times 10^{-3} \mathrm{~s}$ to propagate back and forth through the block and the numerical simulation lasts $0.8 \times 10^{-1}$. By this time, waves are vanishing slowly due to some light numerical damping implemented in the algorithm equivalent to a Rayleigh internal damping. One may estimate that the impact is mild, in the sense that the middle contacting node 16 does not move during the experiment. The final distribution of reaction forces is depicted in Figs. 14, 15. Figure 14 is not significantly different from Fig. 11. However, one may observe that the distribution of forces is no longer symmetric as it was in the quasistatically deformed block. In Fig. 15 the doted
Fig. 13 The quasistatically deformed block at equilibrium and some rigid projectile ready to be thrown horizontally to hit the left side of the block

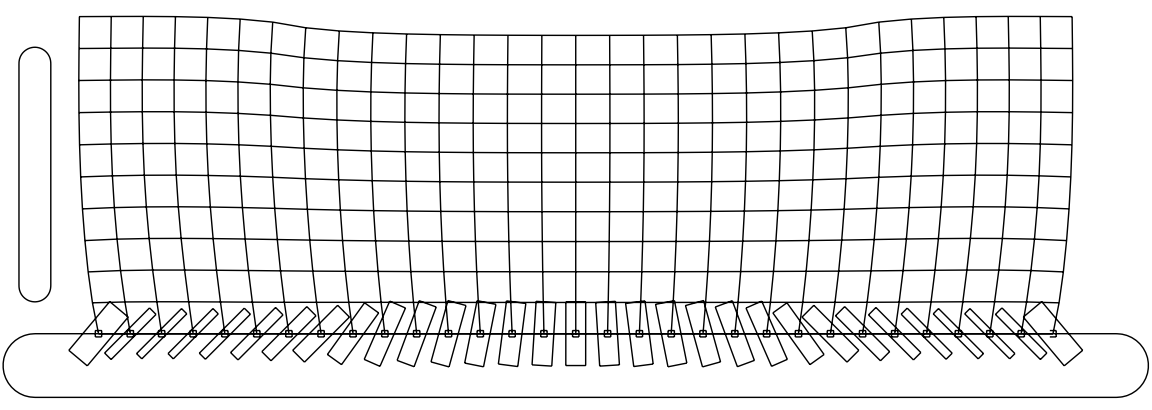

Fig. 14 The settled deformed block after impact. The projectile has been sent back out of the frame

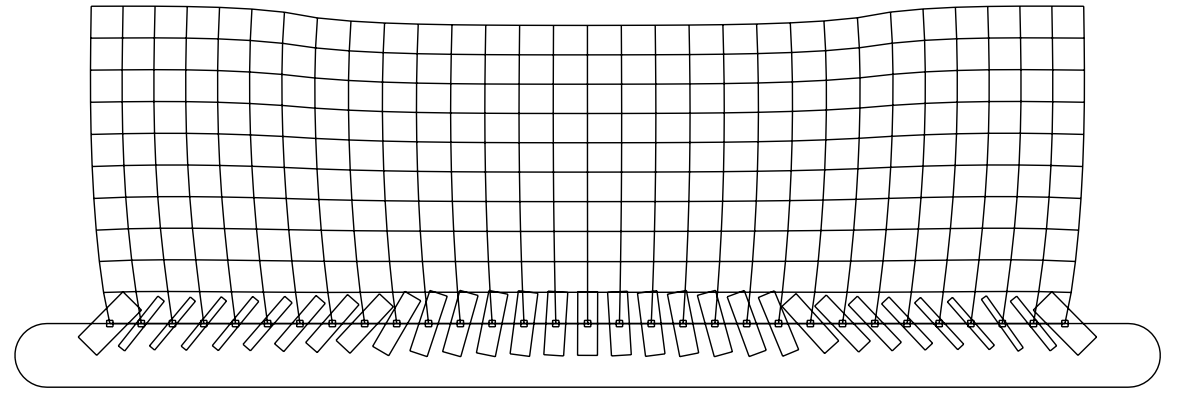


line represents the $\left|R_{t}\right| / \mu R_{n}$ distribution for the quasistatic evolution given in Fig. 12, and the thick line represents the distribution after the impact. The figure shows that imminent slidings have been destroyed and all the reactions on the contact boundary are now strictly inside the cone, though some contacts are still close to sliding. When tracking the status of contacting nodes during the experiment, it may be seen that some imminent sliding contacts slide back and forth before sticking as we observed in many cases on simpler models. In this experiment, the amplitude of the waves is quite smaller near the contact zone where the body is stuck than in the upper layers where the body is free. Nevertheless, micro traveling waves near the contact zone change the status of the contacting nodes to destroy at last all imminent slidings. Since traveling waves are responsible for destroying imminent slidings a numerical experiment using a smaller time step, which would capture higher frequency waves, could

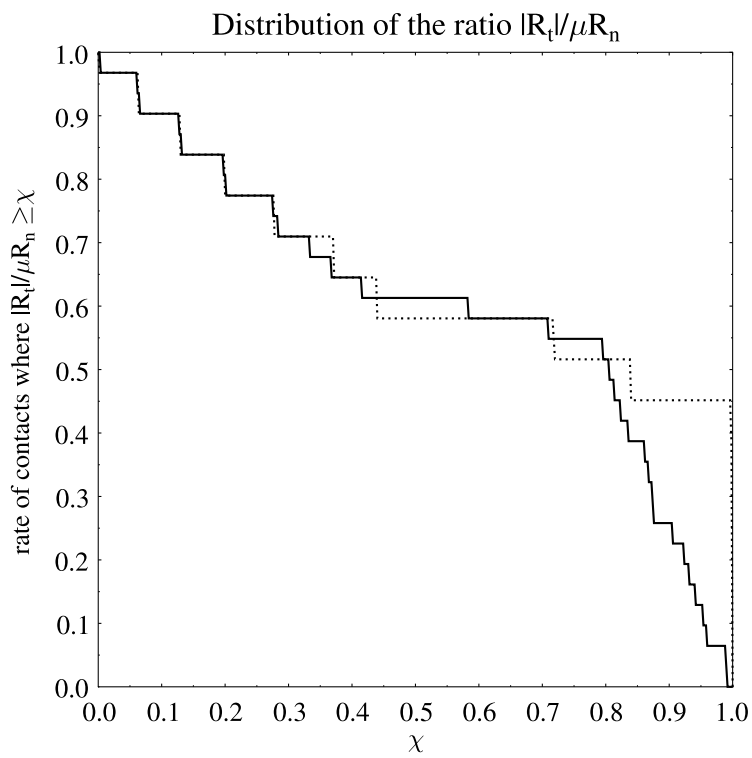

Fig. 15 The $\left|R_{t}\right| / \mu R_{n}$ distribution of the settled impacted block yield a slightly different distribution. But as the time step belongs to the set of parameters of the experiment this would constitute another experiment.

We may note that this experiment emphasizes the fact, reluctantly admitted, that the set of reactions ensuring the equilibrium of a structure, under a given loading, is far from being unique. Indeed, we have obtained here two different distributions of the reaction forces equilibrating the loading: the first one is issued from a quasistatic evolution and the second one is obtained after a dynamical evolution.

This numerical experience furnishes a further justification of the conjecture. Indeed, all the behaviors described in the previous sections have been observed here.

- The main feature is clearly observed: after perturbing an equilibrium state the trajectory leads to a new state where all the reactions are strictly inside the cones. This means, from a mechanical point of view, that starting from an imminent sliding state, the final state is strictly stuck by friction.

- The points which are not in contact but which are only connected to points in contact by an elastic stiffness, continue to oscillate indefinitely, so that a final state where all the points are at equilibrium can be reached only by introducing some damping.

\section{Conclusion}

The stability of equilibrium states of discrete mechanical systems with unilateral contact and nonregularized Coulomb friction has been explored above. We have proposed a conjecture concerning the trajectories obtained by perturbing the external forces in equilibrium states. Having defined a new notion of stability it seems that small perturbations of these external forces tend to increase the stability of the equilibrium states in the following sense. If we explore

Fig. 16 The nodes velocity field at $0.2 \times 10^{-3} \mathrm{~s}$ after the projectile has bounced off the block

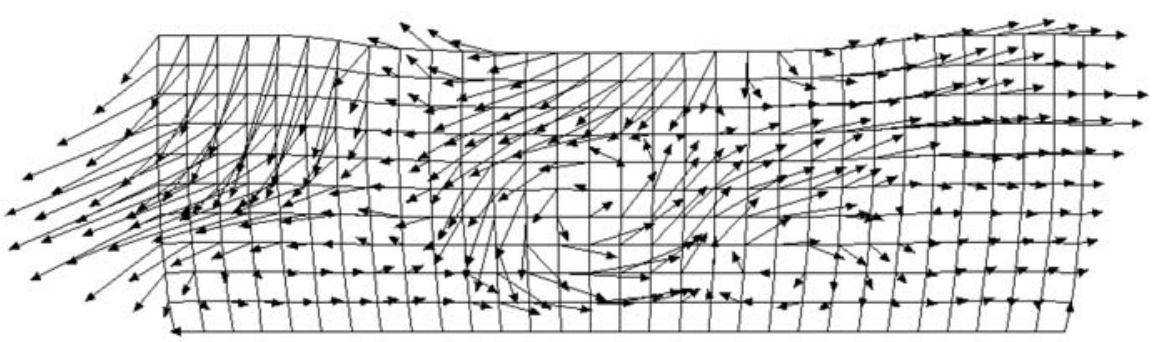


the equilibrium states of systems where all points are strictly in contact with an obstacle then we obtain generically that some points are strictly stuck by friction, whereas others are in imminent sliding. A perturbation of the external forces leads to a trajectory where at least some of the points in imminent sliding are set into motion. We then show that this trajectory leads to a new equilibrium state where the number of points that are strictly stuck by friction is greater than before the perturbation. The system is therefore increasingly stable in the sense that it shall be intuitively more difficult to set the system in motion.

This conjecture has been expressed in more precise terms in the Introduction. We have endeavored throughout this work to back up the conjecture. Although the mathematical tools involved here are basic, they need to be handled with care because of the strong nonlinearities due to the fact that the contact and friction laws are not regularized.

We began by checking that the trajectory of all the particles belonging to a chain of masses sliding on a horizontal plane agrees with the conjecture. We then proceeded to explore the trajectories obtained by perturbing the equilibrium states of a simple model where one mass is in contact with a horizontal plane. This simple model has been extensively studied and our contribution has consisted in showing that the trajectories always agree with the conjecture in particular when different types of oscillating perturbations are applied. Our next step was to consider a model slightly more complicated from a mechanical point of view, two particles instead of one, which turned out to be very much more complicated to analyze. Having explored all the equilibrium states, we have shown that any constant perturbation of the external forces agree with the conjecture. Concerning systems with more degrees of freedom we give the results obtained by a finite element computation and check that they agree exactly with those obtained analytically for the simple models.

This study is of course restricted to discrete systems, and only nonzero stiffness matrices have been considered. Granular media constitute an other important class of discrete systems concerned by this conjecture but it is probable that in this case a more precise definition of the admissible perturbations is necessary. In the meantime a considerable amount of numerical experiments concerning both granular media and other systems with nonzero stiffness matrix are being undergone and shall be published soon.

A direct proof of the conjecture in the general case is for the moment an open problem. We have in this paper simply shown that the conjecture is reasonable. It is however likely that it can be extended to other mechanical systems where the constitutive law is represented by the graph of a multivalued application.

\section{References}

1. Alart, P., Curnier, A.: Contact discret avec frottement: unicité de la solution, convergence de l'algorithme. Publications du Laboratoire de Mécanique Appliquée, Ecole Polytechnique Fédérale de Lausanne (1986)

2. Ballard, P., Léger, A., Pratt, E.: Stability of discrete systems involving shocks and friction. In: Wriggers, P., Nackenhorst, U. (eds.) Analysis and Simulation of Contact Problems. Lecture Notes in Applied and Computational Mechanics, vol. 27, pp. 343-350. Springer, Berlin (2006)

3. Basseville, S., Léger, A.: Stability of equilibrium states in a simple system with unilateral contact and Coulomb friction. Arch. Appl. Mech. 76(7/8), 403-428 (2006)

4. Ballard, P., Basseville, S.: Existence and uniqueness for dynamical unilateral contact with Coulomb friction: a model problem. Math. Model. Numer. Anal. 39(1), 57-77 (2005)

5. Basseville, S., Léger, A., Pratt, E.: Investigation of the equilibrium states and their stability for a simple model with unilateral contact and Coulomb friction. Arch. Appl. Mech. 73, 409-420 (2003)

6. Brezis, H.: Operateurs maximaux monotones et semigroupes de contraction dans les espaces de Hilbert. NorthHolland, Amsterdam (1973)

7. Dubois, F.: LMGC90. http://www.lmgc.univ-montp2.fr

8. Jean, M.: The non-smooth contact dynamics method. Comput. Methods Appl. Mech. Eng. 177, 235-257 (1999)

9. Klarbring, A.: Examples of non-uniqueness and nonexistence of solutions to quasistatic contact problems with friction. Ing. Arch. 60, 529-541 (1990)

10. Martins, J.A.C., Monteiro Marques, M.D.P., Gastaldi, F.: On an example of non-existence of solution to a quasistatic frictional contact problem. Eur. J. Mech. A/Solids 13(1), 113-133 (1994)

11. Moreau, J.J.: Unilateral contact and dry friction in finite freedom dynamics. In: Moreau, J.J., Panagiotopoulos, P.D. (eds.) Nonsmooth Mechanics and Applications. CISM Courses and Lectures, vol. 302. Springer, Vienne (1988)

12. Pinto da Costa, A.M.F.: Instabilidades e bifurcacoes em sistemas de comportamento no-suave. PhD Thesis, Universidade Técnica de Lisboa, Instituto Superior Técnico (2001)

13. Pratt, E., Léger, A., Jean, M.: Critical oscillations of massspring systems due to nonsmooth friction. Arch. Appl. Mech. 78, 89-104 (2008) 